Supporting Information for

\title{
A Useful Method for the Conversion of Olefins to Nitro Olefins
}

\author{
G. Sudhakar Reddy and E. J. Corey* \\ Department of Chemistry and Chemical Biology, Harvard University \\ Cambridge, Massachusetts, 02138 \\ corey@chemistry.harvard.edu
}

Table of Contents

$\begin{array}{ll}\text { 1. General Information } & \text { Page S2 }\end{array}$

2. Nitration of Selected Monocyclic Substrates to form Conjugated Nitro Olefins Page S2

3. Conversion of Trisubstituted Olefins to Allylic Nitro Olefins $\quad$ Page S5

4. Nitration of More Complex Substrates $\quad$ Page S6

$\begin{array}{ll}\text { 5. Nitration-Rearrangement of Cholesteryl Benzoate } & \text { Page S7 }\end{array}$

6. Ortho-Selective Nitration of Conjugated Styrene Derivatives $\quad$ Page S9

$\begin{array}{ll}\text { 7. Para- Selective Nitration } & \text { Page S11 }\end{array}$

$\begin{array}{lr}\text { 8. References } & \text { Page S12 }\end{array}$

$\begin{array}{ll}\text { 9. Copies of }{ }^{1} \mathrm{H} \text { and }{ }^{13} \mathrm{C} \text { NMR Spectra } & \text { Page S14 }\end{array}$ 


\section{General Information:}

Unless stated otherwise, reactions were performed in flame-dried glassware under a positive pressure of nitrogen using anhydrous solvents. $\mathrm{CH}_{2} \mathrm{Cl}_{2}$ and THF were purified by passage through a PPT / Glass Contour Solvent Purification System under argon. Hexane and $\mathrm{Et}_{2} \mathrm{O}$ were freshly distilled from $\mathrm{CaH}_{2}$ before use. Commercial grade reagents and solvents were used without further purification unless otherwise stated. All reactions that require heating were conducted with a water bath/oil bath as the heat source, unless otherwise noted. Thin-layer chromatography (TLC) was performed using Merck silica gel 60 F254 pre-coated plates $(0.25 \mathrm{~mm})$ and visualization was done by $\mathrm{UV}$, or alternatively by $\mathrm{I}_{2}, \mathrm{KMnO}_{4}$, CAM or $p$-anisaldehyde staining. Flash chromatography was performed using Silicycle SiliaFlash F60 (40-63 $\mu \mathrm{m}$ particle size). NMR spectra were recorded in the Laukien- Purcell Instrumentation Center at Harvard University on Varian Spectrometer at the indicated frequency $(400-600 \mathrm{MHz}) .{ }^{1} \mathrm{H}$ and ${ }^{13} \mathrm{C}$ NMR chemical shifts are reported as $\delta$ using residual solvent as an internal standard. High-resolution mass spectral analyses were performed using a Bruker microTOFII ESI spectrometer. IR spectra were measured on Bruker ALPHA FT-IR. Optical rotations were measured with a Anton Paar MCP 150 Modular Compact Polarimeter at the indicated temperature. Melting points (mp) are uncorrected and were recorded on a Fisher- Johns melting point apparatus.

\section{Nitration of Selected Monocyclic Substrates to form Conjugated Nitro Olefins:}

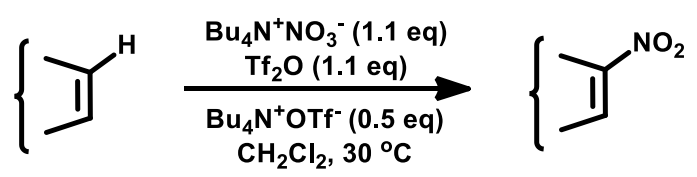

\section{General Procedure for Nitration of Cyclic Olefins (Method A):}

To a stirred solution of tetrabutylammonium nitrate $(3.3 \mathrm{mmol})$ in $\mathrm{CH}_{2} \mathrm{Cl}_{2}(5.0 \mathrm{~mL})$ was added trifluoromethanesulfonic anhydride $(3.3 \mathrm{mmol})$ at $0{ }^{\circ} \mathrm{C}$. The reaction mixture was brought to $30{ }^{\circ} \mathrm{C}$ over $30 \mathrm{~min}$. and then tetrabutylammonium triflate $(1.5 \mathrm{mmol})$ in $2 \mathrm{~mL}$ of $\mathrm{CH}_{2} \mathrm{Cl}_{2}$ was added. The cyclic olefin $(3.0 \mathrm{mmol})$ was then added slowly over $2 \mathrm{~h}$ to this mixture at $30^{\circ} \mathrm{C}$. The resulting solution was stirred for an additional $30 \mathrm{~min}$. at which time starting material has been consumed (monitored by TLC). The reaction mixture was cooled to $0{ }^{\circ} \mathrm{C}$, treated with solid $\mathrm{NaHCO}_{3}(3.0 \mathrm{mmol})$, filtered and concentrated under reduced pressure. The crude product was purified by column chromatography $\left(\mathrm{SiO}_{2}\right.$, hexanes/ethyl acetate) to afford the pure nitro olefin.

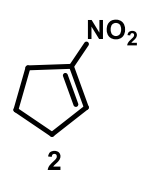




\section{1-Nitrocyclopent-1-ene (2):}

The compound $\mathbf{2}^{1}$ (238 mg) was prepared from cyclopentene (1) (204 mg, $3.0 \mathrm{mmol}$ ) using method A. (70\% yield) after flash chromatography ( $\mathrm{SiO}_{2}$, hexanes/ethyl acetate 19:1) and isolated as a faint yellow liquid. FTIR (neat, $\mathbf{~ c m}^{-1}$ ): 2957, 1507, 1357, 1210, 1142, 905, 721; ${ }^{\mathbf{1}} \mathbf{H} \mathbf{~ N M R}\left(\mathbf{C D C l}_{\mathbf{3}}, \mathbf{4 0 0} \mathbf{M H z}\right): \delta 7.00$ - $6.98(\mathrm{~m}, 1 \mathrm{H}), 2.86-2.79(\mathrm{~m}, 2 \mathrm{H}), 2.64-2.57(\mathrm{~m}, 2 \mathrm{H}), 2.17-2.08(\mathrm{~m}, 2 \mathrm{H}) ;{ }^{13} \mathbf{C}$ NMR $\left(\mathbf{C D C l}_{3}, \mathbf{1 5 0}\right.$ MHz): $\delta$ 138.5, 127.7, 30.9, 29.4, 22.1; MS (ESI) m/z: $\mathrm{C}_{5} \mathrm{H}_{7} \mathrm{NO}_{2}[\mathrm{M}+\mathrm{H}]^{+} 114.0$.

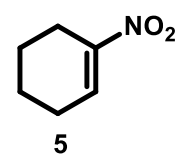

\section{1-Nitrocyclohex-1-ene (5):}

The compound $\mathbf{5}^{2}(297 \mathrm{mg}$ ) was prepared from cyclohexene (4) $(246 \mathrm{mg}, 3.0 \mathrm{mmol})$ using method A. (78\% yield) after flash chromatography $\left(\mathrm{SiO}_{2}\right.$, hexanes/ethyl acetate 19:1) and isolated as a faint yellow oil. FTIR (neat, $\left.\mathbf{c m}^{-1}\right)$ : 2943, 2868, 1667, 1514, 1444, 1336, 1058, 904, 728; ${ }^{\mathbf{1}} \mathbf{H}$ NMR (CDCl 3 , 400 MHz): $\delta 7.33-7.30(\mathrm{~m}, 1 \mathrm{H}), 2.59-2.55(\mathrm{~m}, 2 \mathrm{H}), 2.34-2.30(\mathrm{~m}, 2 \mathrm{H}), 1.80-1.74(\mathrm{~m}, 2 \mathrm{H}), 1.66-1.60$ $(\mathrm{m}, 2 \mathrm{H}) ;{ }^{13} \mathbf{C ~ N M R}\left(\mathbf{C D C l}_{3}, \mathbf{1 5 0} \mathbf{M H z}\right): \delta 149.7,134.3,24.8,24.0,21.8,20.7 ; \mathbf{H R M S}(\mathbf{E S I}) \mathbf{m} / \mathbf{z}:[\mathrm{M}+$ $\mathrm{H}]^{+}$Calcd for $\mathrm{C}_{6} \mathrm{H}_{10} \mathrm{NO}_{2}$ 128.0706; Found 128.0704.

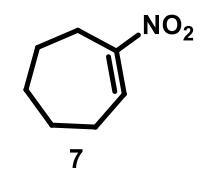

\section{1-Nitrocyclohept-1-ene (7):}

The compound $7^{2}$ (318 mg) was prepared from cycloheptene (6) (288 mg, $3.0 \mathrm{mmol}$ ) using method A. (75\% yield) after flash chromatography ( $\mathrm{SiO}_{2}$, hexanes/ethyl acetate 19:1) and isolated as a colorless oil.

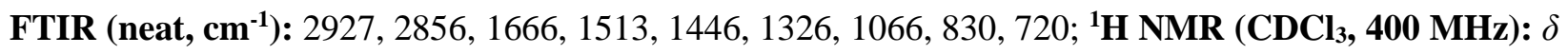
$7.26(\mathrm{t}, J=6.7 \mathrm{~Hz}, 1 \mathrm{H}), 2.73-2.66(\mathrm{~m}, 2 \mathrm{H}), 2.22-2.14(\mathrm{~m}, 2 \mathrm{H}), 1.65-1.58(\mathrm{~m}, 2 \mathrm{H}), 1.53-1.45(\mathrm{~m}$, 4H); ${ }^{13} \mathbf{C ~ N M R}\left(\mathbf{C D C l}_{3}, \mathbf{1 5 0} \mathbf{M H z}\right): \delta 155.3,137.7,30.4,27.6,26.5,25.4,25.1 ; \mathbf{H R M S}$ (ESI) m/z: [M + $\mathrm{H}]^{+}$Calcd for $\mathrm{C}_{7} \mathrm{H}_{12} \mathrm{NO}_{2}$ 142.0863; Found 142.0868.

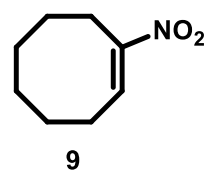

\section{1-Nitrocyclooct-1-ene (9):}

The compound $\mathbf{9}^{\mathrm{b}}$ (359 mg) was prepared from cyclooctene $(\mathbf{8})$ (330 mg, $3.0 \mathrm{mmol}$ ) using method A. (77\% yield) after flash chromatography $\left(\mathrm{SiO}_{2}\right.$, hexanes/ethyl acetate 19:1) and isolated as a colorless oil. FTIR (neat, $\left.\mathbf{c m}^{-1}\right)$ : 2928, 2858, 1664, 1516, 1456, 1332, 1008, 827, 745; ${ }^{1} \mathbf{H}$ NMR (CDCl 3 , 400 MHz): $\delta$ 
$7.30(\mathrm{t}, J=8.8 \mathrm{~Hz}, 1 \mathrm{H}), 2.77-2.72(\mathrm{~m}, 2 \mathrm{H}), 2.36-2.29(\mathrm{~m}, 2 \mathrm{H}), 1.76-1.68(\mathrm{~m}, 4 \mathrm{H}), 1.55-1.48(\mathrm{~m}$,

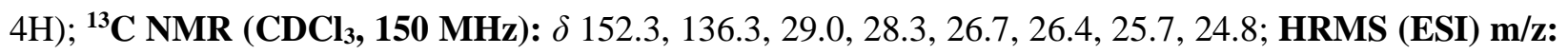
$[\mathrm{M}+\mathrm{H}]^{+}$Calcd for $\mathrm{C}_{8} \mathrm{H}_{14} \mathrm{NO}_{2}$ 156.1019; Found 156.1022.

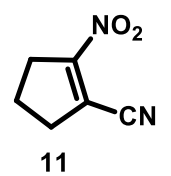

2-Nitrocyclopent-1-enecarbonitrile (11):

The compound $\mathbf{1 1}^{3}$ (332 mg) was prepared from 1-cyanocyclopentene (10) (279 mg, $3.0 \mathrm{mmol}$ ) using method A. (80\% yield) after flash chromatography $\left(\mathrm{SiO}_{2}\right.$, hexanes/ethyl acetate $\left.4: 1\right)$ and isolated as a

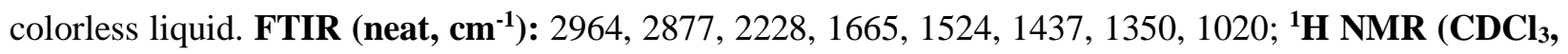
400 MHz): $\delta 3.10-3.03(\mathrm{~m}, 2 \mathrm{H}), 2.98-2.91(\mathrm{~m}, 2 \mathrm{H}), 2.22(\mathrm{p}, J=7.9 \mathrm{~Hz}, 2 \mathrm{H}) ;{ }^{13} \mathbf{C} \mathbf{~ N M R}\left(\mathbf{C D C l}_{3}, \mathbf{1 0 0}\right.$ MHz): $\delta$ 160.4, 117.3, 112.4, 34.6, 31.1, 20.6; HRMS (ESI) m/z: $[\mathrm{M}+\mathrm{H}]^{+}$Calcd for $\mathrm{C}_{6} \mathrm{H}_{7} \mathrm{~N}_{2} \mathrm{O}_{2}$ 139.0502; Found 139.0507.

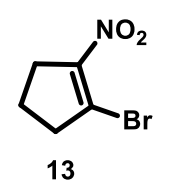

\section{1-Bromo-2-nitrocyclopent-1-ene (13):}

The compound $\mathbf{1 3}^{2}$ (427 mg) was prepared from 1-bromocyclopentene (12) (441 mg, $3.0 \mathrm{mmol}$ ) using method A. (74\% yield) after flash chromatography $\left(\mathrm{SiO}_{2}\right.$, hexanes/ethyl acetate 9:1) and isolated as a faint yellow liquid. FTIR (neat, $\left.\mathbf{c m}^{-1}\right):$ 2959, 2859, 1616, 1511, 1340, 1075; ${ }^{1} \mathbf{H}$ NMR (CDCl, 400 MHz): $\delta 2.94(\mathrm{t}, J=7.7 \mathrm{~Hz}, 4 \mathrm{H}), 2.13-2.05(\mathrm{~m}, 2 \mathrm{H}) ;{ }^{13} \mathbf{C}$ NMR $\left(\mathbf{C D C l}_{3}, \mathbf{1 0 0} \mathbf{M H z}\right): \delta 148.3,128.4$, 41.3, 31.4, 20.0; HRMS (ESI) m/z: $[\mathrm{M}+\mathrm{H}]^{+}$Calcd for $\mathrm{C}_{5} \mathrm{H}_{7} \mathrm{BrNO}_{2}$ 193.0874; Found 193.0880.

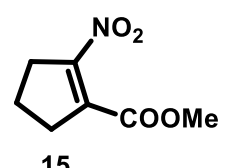

\section{Methyl 2-nitrocyclopent-1-enecarboxylate (15):}

The compound 15 (380 mg) was prepared from methyl cyclopent-1-enecarboxylate (14) (378 mg, 3.0 mmol) using method A. (74\% yield) after flash chromatography $\left(\mathrm{SiO}_{2}\right.$, hexanes/ethyl acetate 4:1) and isolated as a colorless liquid. FTIR (neat, $\mathbf{c m}^{-1}$ ): 2956, 2853, 1736, 1665, 1519, 1437, 1357, 1098; ${ }^{1} \mathbf{H}$ NMR (CDCl 3 , 400 MHz): $\delta 3.84$ (s, 3H), $3.01-2.95$ (m, 2H), $2.89-2.83$ (m, 2H), $2.17-2.10$ (m, 2H);

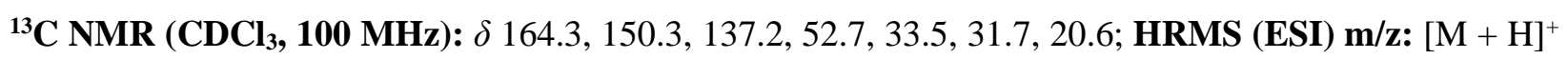
Calcd for $\mathrm{C}_{7} \mathrm{H}_{10} \mathrm{NO}_{4}$ 172.0604; Found 172.0608. 


\section{Conversion of Trisubstituted Olefins to Allylic Nitro Olefins:}
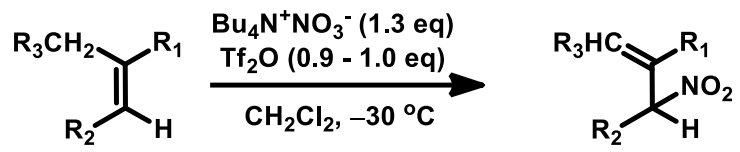

\section{General Procedure for Synthesis of Allylic Nitro Olefins (Method B):}

To a stirred solution of trisubstituted olefin $(3.0 \mathrm{mmol})$ and tetrabutylammonium nitrate $(3.9 \mathrm{mmol})$ in $\mathrm{CH}_{2} \mathrm{Cl}_{2}(5.0 \mathrm{~mL})$ was added trifluoromethanesulfonic anhydride $(2.7-3.0 \mathrm{mmol})$ slowly over $30 \mathrm{~min}$. at $-30{ }^{\circ} \mathrm{C}$. The resulting solution was stirred for an additional $30 \mathrm{~min}$. at which time starting material has been consumed (monitored by TLC). The reaction mixture was treated with solid $\mathrm{NaHCO}_{3}(3.0 \mathrm{mmol})$, filtered and concentrated under reduced pressure. The crude product was purified by column chromatography $\left(\mathrm{SiO}_{2}\right.$, hexanes/ethyl acetate) to afford the pure allylic nitro olefin.

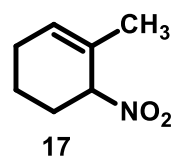

\section{1-Methyl-6-nitrocyclohex-1-ene (17):}

The compound $\mathbf{1 7}^{4}$ (356 mg) was prepared from 1-methylcyclohex-1-ene (16) (288 mg, $3.0 \mathrm{mmol}$ ) using method B. (84\% yield) after flash chromatography $\left(\mathrm{SiO}_{2}\right.$, hexanes/ethyl acetate 19:1) and isolated as a colorless liquid. FTIR (neat, $\left.\mathbf{c m}^{-1}\right): 2939,2861,1635,1553,1341,1280,823 ;{ }^{1} \mathbf{H}_{\text {NMR }}\left(\mathbf{C D C l}_{3}, 400\right.$ MHz): $\delta 5.92-5.84(\mathrm{~m}, 1 \mathrm{H}), 4.91-4.86(\mathrm{~m}, 1 \mathrm{H}), 2.35-2.12(\mathrm{~m}, 2 \mathrm{H}), 2.07-2.00(\mathrm{~m}, 2 \mathrm{H}), 1.72(\mathrm{~s}, 3 \mathrm{H})$, 1.67 - 1.57 (m, 2H); ${ }^{13} \mathbf{C}$ NMR ( $\left.\mathbf{C D C l}_{3}, \mathbf{1 0 0} \mathbf{M H z}\right): \delta 131.1,126.6,85.7,28.5,24.7,20.8,17.8$; HRMS (ESI) $\mathbf{m} / \mathbf{z :}[\mathrm{M}+\mathrm{H}]^{+}$Calcd for $\mathrm{C}_{7} \mathrm{H}_{12} \mathrm{NO}_{2}$ 142.0863; Found 142.0864.

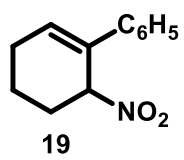

\section{6-Nitro-1-phenylcyclohexene (19):}

The compound $\mathbf{1 9}^{\mathrm{1b}}$ (500 mg) was prepared from 1-phenylcyclohex-1-ene (18) (474 mg, $3.0 \mathrm{mmol}$ ) using method B. (82\% yield) after flash chromatography $\left(\mathrm{SiO}_{2}\right.$, hexanes/ethyl acetate 19:1) and isolated as a faint yellow oil. FTIR (neat, $\mathbf{c m}^{-1}$ ): 3038, 2944, 1600, 1543, 1515, 1447, 1357, 1156, 1070, 768; ${ }^{1} \mathbf{H}$ NMR $\left(\mathbf{C D C l}_{3}, 400 \mathrm{MHz}\right): \delta 7.30-7.19(\mathrm{~m}, 5 \mathrm{H}), 6.41(\mathrm{t}, J=4.4 \mathrm{~Hz}, 1 \mathrm{H}), 5.58-5.52(\mathrm{~m}, 1 \mathrm{H}), 2.47-$ $2.33(\mathrm{~m}, 2 \mathrm{H}), 2.28-2.09$ (m, 2H), $1.81-1.68$ (m, 2H); ${ }^{\mathbf{1 3}} \mathbf{C} \mathbf{~ N M R}\left(\mathbf{C D C l}_{\mathbf{3}}, \mathbf{1 0 0} \mathbf{M H z}\right): \delta 138.8,133.8$, 131.3, 128.7, 127.7, 125.5, 83.0, 29.0, 25.4, 17.3; HRMS (ESI) m/z: $[\mathrm{M}+\mathrm{H}]^{+}$Calcd for $\mathrm{C}_{12} \mathrm{H}_{14} \mathrm{NO}_{2}$ 204.1019; Found 204.1023. 


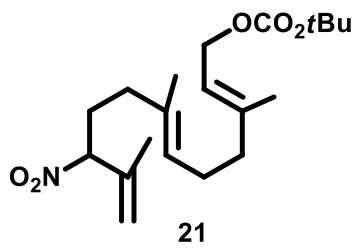

tert-Butyl ((2E,6E)-3,7,11-trimethyl-10-nitrododeca-2,6,11-trien-1-yl) carbonate (21):

The compound 21 (771 mg) was prepared from E,E-farnesol BOC ester (20) (966 mg, $3.0 \mathrm{mmol}$ ) using method B. (70\% yield) after flash chromatography $\left(\mathrm{SiO}_{2}\right.$, hexanes/ethyl acetate 49:1) and isolated as a

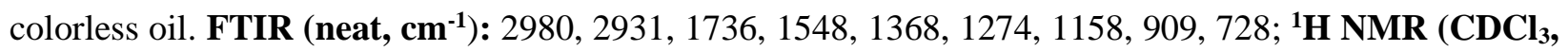
$400 \mathrm{MHz}): \delta 5.36(\mathrm{t}, J=7.4 \mathrm{~Hz}, 1 \mathrm{H}), 5.18-5.09(\mathrm{~m}, 3 \mathrm{H}), 4.90-4.84(\mathrm{~m}, 1 \mathrm{H}), 4.58(\mathrm{~d}, J=7.1 \mathrm{~Hz}, 2 \mathrm{H})$, $2.34-2.24(\mathrm{~m}, 1 \mathrm{H}), 2.15-2.07(\mathrm{~m}, 2 \mathrm{H}), 2.06-2.01(\mathrm{~m}, 2 \mathrm{H}), 2.00-1.88(\mathrm{~m}, 3 \mathrm{H}), 1.80(\mathrm{~s}, 3 \mathrm{H}), 1.70(\mathrm{~s}$, 3H), 1.60 (s, 3H), 1.48 (s, 9H); ${ }^{13} \mathbf{C}$ NMR (CDCl 3 , 100 MHz): $\delta 153.6,142.1,138.7,132.9,125.8,118.6$, 118.3, 92.2, 81.9, 63.7, 39.2, 35.6, 29.2, 27.8, 26.1, 18.2, 16.5, 15.8; HRMS (ESI) m/z: $[\mathrm{M}+\mathrm{Na}]^{+} \mathrm{Calcd}$ for $\mathrm{C}_{20} \mathrm{H}_{33} \mathrm{NNaO}_{5}$ 390.2251; Found 390.2253.

\section{Nitration of More Complex Substrates:}

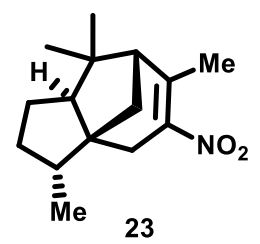

$(3 R, 3 \mathrm{a} R, 7 R, 8 \mathrm{a} S)-3,6,8,8$-Tetramethyl-5-nitro-2,3,4,7,8,8a-hexahydro-1H-3a,7-methanoazulene (23): The compound 23 (635 mg) was prepared from cedrene (22) (612 mg, $3.0 \mathrm{mmol}$ ) using method A. (85\% yield) after flash chromatography $\left(\mathrm{SiO}_{2}\right.$, hexanes/ethyl acetate 19:1) and isolated as a colorless oil. FTIR (neat, $\left.\mathbf{c m}^{-1}\right)$ : 2953, 2875, 1641, 1508, 1465, 1362, 975, 768; ${ }^{\mathbf{1}} \mathbf{H}$ NMR (CDCl $\left.\mathbf{3}, 600 \mathrm{MHz}\right): \delta 2.72(\mathrm{dq}, J$ $=16.5,2.2 \mathrm{~Hz}, 1 \mathrm{H}), 2.46(\mathrm{dt}, J=16.5,1.7 \mathrm{~Hz}, 1 \mathrm{H}), 2.15(\mathrm{t}, J=2.0 \mathrm{~Hz}, 3 \mathrm{H}), 2.13(\mathrm{~d}, J=3.6 \mathrm{~Hz}, 1 \mathrm{H}), 1.93$ - $1.86(\mathrm{~m}, 2 \mathrm{H}), 1.79-1.73(\mathrm{~m}, 2 \mathrm{H}), 1.69-1.63(\mathrm{~m}, 1 \mathrm{H}), 1.48-1.41(\mathrm{~m}, 3 \mathrm{H}), 1.05(\mathrm{~s}, 3 \mathrm{H}), 1.02(\mathrm{~s}, 3 \mathrm{H})$, $0.92(\mathrm{~d}, J=7.0 \mathrm{~Hz}, 3 \mathrm{H}) ;{ }^{13} \mathbf{C} \mathbf{~ N M R}\left(\mathbf{C D C l}_{3}, \mathbf{1 5 0} \mathbf{~ M H z}\right): \delta 148.7,144.2,58.9,57.9,54.1,49.2,41.1,39.8$, 39.1, 36.0, 27.7, 25.9, 24.8, 23.5, 15.4; HRMS (ESI) m/z: $[\mathrm{M}+\mathrm{Na}]^{+}$Calcd for $\mathrm{C}_{15} \mathrm{H}_{23} \mathrm{NNaO}_{2} 272.1621$; Found 272.1622; Optical rotation: $[\boldsymbol{\alpha}]_{\mathrm{D}}{ }^{23}=-101.6\left(c 1.0, \mathrm{CHCl}_{3}\right)$. 


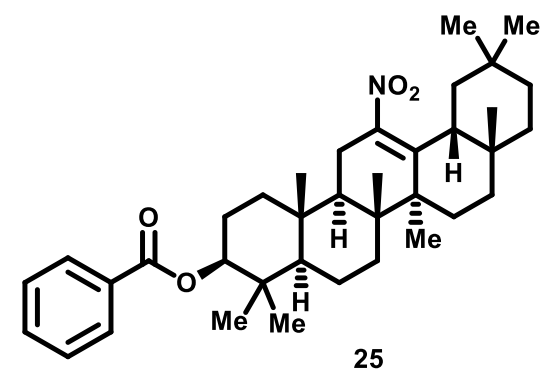

$(3 S, 4 \mathrm{a} R, 6 \mathrm{a} R, 6 \mathrm{~b} S, 8 \mathrm{a} R, 12 \mathrm{a} R, 14 \mathrm{a} R, 14 \mathrm{~b} R)-4,4,6 \mathrm{a}, 6 \mathrm{~b}, 8 \mathrm{a}, 11,11,14 \mathrm{~b}-O c t a m e t h y l-13-n i t r o-1,2,3,4,4 \mathrm{a}, 5,6$, 6a,6b,7,8,8a,9,10,11,12,12a,14,14a,14b-icosahydropicen-3-yl benzoate (25):

The compound 25 (1.40 g) was prepared from $\beta$-amyrin benzoate (24) (1.59 g, $3.0 \mathrm{mmol})$ using method A. (81\% yield) after flash chromatography $\left(\mathrm{SiO}_{2}\right.$, hexanes/ethyl acetate 19:1) and isolated as a white solid. M. P: $221-222{ }^{\circ}$ C; FTIR (neat, $\mathbf{c m}^{-1}$ ): 2948, 2861, 1714, 1603, 1515, 1459, 1274, 1112, 910, 734; ${ }^{1} \mathbf{H}$ NMR ( $\left.\mathbf{C D C l}_{3}, 400 \mathrm{MHz}\right): \delta 8.06-8.02(\mathrm{~m}, 2 \mathrm{H}), 7.58-7.53(\mathrm{~m}, 1 \mathrm{H}), 7.47-7.42(\mathrm{~m}, 2 \mathrm{H}), 4.76$ (dd, $J=11.2,5.2 \mathrm{~Hz}, 1 \mathrm{H}), 2.57$ (dd, $J=17.7,11.8 \mathrm{~Hz}, 1 \mathrm{H}), 2.43-2.29$ (m, 2H), $2.09-2.02(\mathrm{~m}, 1 \mathrm{H})$, $1.94-1.76(\mathrm{~m}, 3 \mathrm{H}), 1.74-1.60(\mathrm{~m}, 4 \mathrm{H}), 1.58-1.51(\mathrm{~m}, 2 \mathrm{H}), 1.50-1.26(\mathrm{~m}, 6 \mathrm{H}), 1.25(\mathrm{~s}, 3 \mathrm{H}), 1.23-$ $1.10(\mathrm{~m}, 4 \mathrm{H}), 1.08$ (s, 3H), $1.06(\mathrm{~s}, 3 \mathrm{H}), 1.04(\mathrm{~s}, 3 \mathrm{H}), 0.97$ (s, 3H), $0.91(\mathrm{~s}, 3 \mathrm{H}), 0.87$ (s, 3H), $0.83(\mathrm{~s}, 3 \mathrm{H})$;

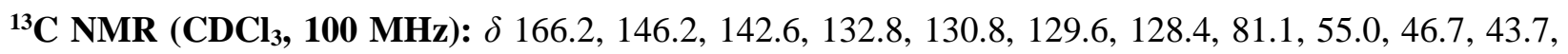
41.9, 41.3, 40.2, 38.2, 38.1, 37.0, 36.9, 34.6, 33.1, 32.4, 32.1, 31.2, 28.2, 28.2, 26.3, 26.2, 25.8, 25.6, 23.5, 23.1, 18.0, 17.0, 16.7, 15.6; HRMS (ESI) m/z: $[\mathrm{M}+\mathrm{Na}]^{+}$Calcd for $\mathrm{C}_{37} \mathrm{H}_{53} \mathrm{NNaO}_{4}$ 598.3867; Found 598.3872; Optical rotation: $[\boldsymbol{\alpha}]_{\mathbf{D}}{ }^{23}=+124.3\left(c 1.0, \mathrm{CHCl}_{3}\right)$.

\section{Nitration-Rearrangement of Cholesteryl Benzoate:}

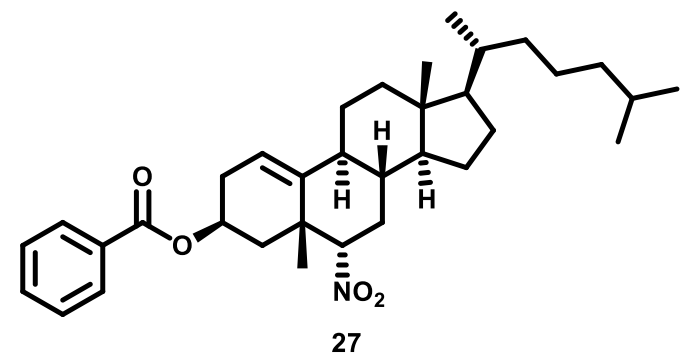

(3S,5R,6S,8S,9S,13R,14S,17R)-5,13-Dimethyl-17-((R)-6-methylheptan-2-yl)-6-nitro-3,4,5,6,7,8,9,11, 12,13,14,15,16,17-tetradecahydro- $2 H$-cyclopenta[ $a]$ phenanthren-3-yl benzoate (27):

The compound 27 (1.22 g) was prepared from cholesteryl benzoate (26) (1.47 g, $3.0 \mathrm{mmol})$ using method A. (76\% yield) after flash chromatography $\left(\mathrm{SiO}_{2}\right.$, hexanes/ethyl acetate 19:1) and isolated as a colorless oil. FTIR (neat, $\left.\mathbf{c m}^{-1}\right)$ : 2946, 2867, 1717, 1611, 1548, 1458, 1372, 1272, 1109, 713; ${ }^{1} \mathbf{H} \mathbf{N M R}\left(\mathbf{C D C l}_{3}\right.$, $400 \mathrm{MHz}): \delta 8.03-7.98(\mathrm{~m}, 2 \mathrm{H}), 7.58-7.53(\mathrm{~m}, 1 \mathrm{H}), 7.46-7.40(\mathrm{~m}, 2 \mathrm{H}), 5.53(\mathrm{dt}, J=5.4,2.7 \mathrm{~Hz}$, $1 \mathrm{H}), 5.43-5.37(\mathrm{~m}, 1 \mathrm{H}), 4.65$ (t, $J=8.6 \mathrm{~Hz}, 1 \mathrm{H}), 2.79$ (dddd, $J=19.0,7.9,5.3,2.7 \mathrm{~Hz}, 1 \mathrm{H}), 2.49$ (ddd, $J$ 
= 13.8, 9.2, 7.5 Hz, 1H), $2.39-2.32(\mathrm{~m}, 1 \mathrm{H}), 2.06-2.01(\mathrm{~m}, 1 \mathrm{H}), 1.96-1.94(\mathrm{~m}, 1 \mathrm{H}), 1.91-1.82(\mathrm{~m}$, $3 \mathrm{H}), 1.82-1.71(\mathrm{~m}, 2 \mathrm{H}), 1.65-1.60(\mathrm{~m}, 1 \mathrm{H}), 1.56-1.49(\mathrm{~m}, 2 \mathrm{H}), 1.44(\mathrm{~s}, 3 \mathrm{H}), 1.42-1.30(\mathrm{~m}, 5 \mathrm{H})$, $1.28-1.22(\mathrm{~m}, 2 \mathrm{H}), 1.19-1.08(\mathrm{~m}, 6 \mathrm{H}), 0.92(\mathrm{~d}, J=6.5 \mathrm{~Hz}, 3 \mathrm{H}), 0.88(\mathrm{~d}, J=1.9 \mathrm{~Hz}, 3 \mathrm{H}), 0.86(\mathrm{~d}, J=$ $2.0 \mathrm{~Hz}, 3 \mathrm{H}), 0.70(\mathrm{~s}, 3 \mathrm{H}) ;{ }^{13} \mathbf{C}$ NMR $\left(\mathbf{C D C l}_{3}, \mathbf{1 0 0} \mathbf{M H z}\right): \delta$ 166.0, 143.8, 133.0, 130.5, 129.6, 128.4, 118.3, 90.9, 67.6, 57.0, 55.9, 42.9, 42.2, 40.4, 39.5, 39.4, 38.4, 36.1, 35.7, 33.2, 30.6, 30.1, 28.1, 28.0, 27.1, 23.8, 23.6, 22.8, 22.7, 22.6, 18.7, 12.1; HRMS (ESI) m/z: $[\mathrm{M}+\mathrm{H}]^{+}$Calcd for $\mathrm{C}_{34} \mathrm{H}_{50} \mathrm{NO}_{4}$ 536.3734; Found 536.3744; Optical rotation: $[\alpha]_{\mathbf{D}}{ }^{23}=-28.7\left(\right.$ c 1.0, $\left.\mathrm{CHCl}_{3}\right)$.

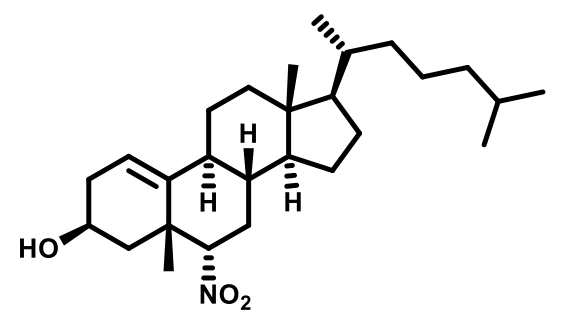

28

(3S,5R,6S,8S,9S,13R,14S,17R)-5,13-Dimethyl-17-((R)-6-methylheptan-2-yl)-6-nitro-3,4,5,6,7,8,9,11, $12,13,14,15,16,17$-tetradecahydro-2 $H$-cyclopenta[ $[a]$ phenanthren-3-ol (28):

To a stirred solution of compound $27(100 \mathrm{mg}, 0.2 \mathrm{mmol})$ in $\mathrm{MeOH}(1.0 \mathrm{~mL})$ was added potassium hydroxide $(52 \mathrm{mg}, 1.0 \mathrm{mmol})$ at room temperature. After heating for $3 \mathrm{~h}$ at $50{ }^{\circ} \mathrm{C}$ the reaction was complete as judged by TLC analysis. The reaction mixture was cooled to room temperature, filtered and evaporated under reduced pressure to give the crude product. Purification of the crude compound by column chromatography $\left(\mathrm{SiO}_{2}\right.$, hexanes/ethyl acetate 9:1) provided the alcohol 28 (81 $\mathrm{mg}, 94 \%$ yield) as a colorless oil.

FTIR (neat, $\left.\mathbf{c m}^{-1}\right)$ : 3369, 2942, 2867, 1546, 1461, 1370, 1043, 753; ${ }^{1} \mathbf{H}_{\text {NMR }}\left(\mathbf{C D C l}_{3}, \mathbf{4 0 0} \mathbf{~ M H z}\right): \delta 5.51$ $(\mathrm{dt}, J=5.5,2.6 \mathrm{~Hz}, 1 \mathrm{H}), 4.59(\mathrm{t}, J=7.9 \mathrm{~Hz}, 1 \mathrm{H}), 4.09(\mathrm{dq}, J=6.6,5.1 \mathrm{~Hz}, 1 \mathrm{H}), 2.54(\mathrm{dtd}, J=17.8,6.2$, $2.3 \mathrm{~Hz}, 1 \mathrm{H}), 2.40(\mathrm{dt}, J=14.3,7.2 \mathrm{~Hz}, 1 \mathrm{H}), 2.17-2.10(\mathrm{~m}, 1 \mathrm{H}), 2.06-1.94(\mathrm{~m}, 3 \mathrm{H}), 1.93-1.78(\mathrm{~m}$, $3 \mathrm{H}), 1.77-1.70(\mathrm{~m}, 1 \mathrm{H}), 1.64-1.57(\mathrm{~m}, 3 \mathrm{H}), 1.54-1.46(\mathrm{~m}, 3 \mathrm{H}), 1.44-1.40(\mathrm{~m}, 1 \mathrm{H}), 1.35(\mathrm{~s}, 3 \mathrm{H})$, $1.31-1.28(\mathrm{~m}, 1 \mathrm{H}), 1.27-1.20(\mathrm{~m}, 2 \mathrm{H}), 1.16-1.08(\mathrm{~m}, 5 \mathrm{H}), 1.06-0.95(\mathrm{~m}, 2 \mathrm{H}), 0.91$ (d, J = $6.5 \mathrm{~Hz}$, $3 \mathrm{H}), 0.87(\mathrm{~d}, J=1.9 \mathrm{~Hz}, 3 \mathrm{H}), 0.86(\mathrm{~d}, J=1.9 \mathrm{~Hz}, 3 \mathrm{H}), 0.70(\mathrm{~s}, 3 \mathrm{H}) ;{ }^{\mathbf{1 3}} \mathbf{C} \mathbf{N M R}\left(\mathbf{C D C l}_{3}, \mathbf{1 0 0} \mathbf{M H z}\right): \delta$ 143.2, 119.7, 90.8, 64.7, 56.8, 56.0, 44.1, 43.8, 43.2, 39.7, 39.5, 39.3, 36.2, 35.7, 33.6, 33.1, 30.4, 28.2, 28.0, 27.9, 23.8, 23.6, 23.5, 22.8, 22.6, 18.7, 12.3; HRMS (ESI) m/z: $[\mathrm{M}+\mathrm{Na}]^{+}$Calcd for $\mathrm{C}_{27} \mathrm{H}_{45} \mathrm{NNaO}_{3}$ 454.3292; Found 454.3297; Optical rotation: $[\boldsymbol{\alpha}]_{\mathbf{D}}{ }^{23}=-17.3\left(c 1.0, \mathrm{CHCl}_{3}\right)$. 


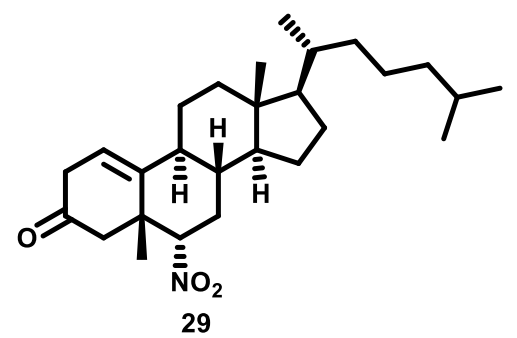

$(5 R, 6 S, 8 S, 9 S, 13 R, 14 S, 17 R)-5,13-D i m e t h y l-17-((R)-6-m e t h y l h e p t a n-2-y l)-6-n i t r o-5,6,7,8,9,11,12,13$, 14,15,16,17-dodecahydro-2H-cyclopenta[a]phenanthren-3(4H)-one (29):

To a stirred solution of pyridinium chlorochromate $(43 \mathrm{mg}, 0.2 \mathrm{mmol})$ in methylene chloride $(0.5 \mathrm{~mL})$ was added alcohol 28 (50 mg, $0.1 \mathrm{mmol}$ in $0.5 \mathrm{~mL}$ of $\left.\mathrm{CH}_{2} \mathrm{Cl}_{2}\right)$ at room temperature. The solution became briefly homogeneous before depositing the brown insoluble reduced reagent. After stirring for $2 \mathrm{~h}$ at room temperature the oxidation was complete as judged by TLC. The brown reaction mixture was diluted with anhydrous ether, the solvent was decanted, and the brown solid was washed twice with diethyl ether. The solvent was evaporated and the crude product was purified by column chromatography $\left(\mathrm{SiO}_{2}\right.$, hexanes/ethyl acetate 19:1) to obtained the ketone $\mathbf{2 9}(39 \mathrm{mg}, 91 \%)$ as a colorless oil.

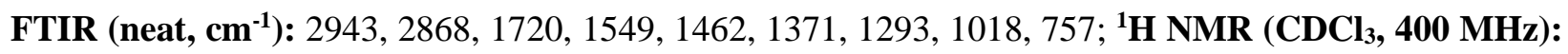
$\delta 5.63(\mathrm{dt}, J=4.5,3.0 \mathrm{~Hz}, 1 \mathrm{H}), 4.78(\mathrm{dd}, J=11.2,7.9 \mathrm{~Hz}, 1 \mathrm{H}), 2.96(\mathrm{td}, J=3.4,1.7 \mathrm{~Hz}, 2 \mathrm{H}), 2.58-2.48$ (m, 2H), $2.28(\mathrm{~d}, J=14.7 \mathrm{~Hz}, 1 \mathrm{H}), 2.06(\mathrm{dt}, J=12.8,3.3 \mathrm{~Hz}, 1 \mathrm{H}), 1.96-1.84(\mathrm{~m}, 4 \mathrm{H}), 1.79-1.71(\mathrm{~m}$, $1 \mathrm{H}), 1.66-1.60(\mathrm{~m}, 1 \mathrm{H}), 1.56-1.49(\mathrm{~m}, 2 \mathrm{H}), 1.40-1.32(\mathrm{~m}, 4 \mathrm{H}), 1.31-1.25(\mathrm{~m}, 2 \mathrm{H}), 1.20(\mathrm{~d}, J=1.2$ $\mathrm{Hz}, 3 \mathrm{H}), 1.17-1.09$ (m, 6H), $0.92(\mathrm{~d}, J=6.5 \mathrm{~Hz}, 3 \mathrm{H}), 0.87$ (d, $J=1.9 \mathrm{~Hz}, 3 \mathrm{H}), 0.86$ (d, J = 1.9 Hz, 3H), 0.68 (s, 3H); ${ }^{13} \mathbf{C}$ NMR (CDCl $\mathbf{3}, \mathbf{1 0 0} \mathbf{~ M H z ) : ~} \delta$ 207.9, 145.2, 117.1, 89.2, 57.3, 55.9, 50.9, 42.7, 41.0, 40.3, 39.5, 39.1, 38.9, 36.1, 35.7, 33.3, 29.4, 28.0, 28.0, 25.7, 23.8, 23.7, 22.8, 22.6, 20.9, 18.7, 11.8; HRMS

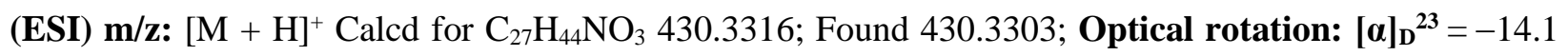
(c $\left.1.0, \mathrm{CHCl}_{3}\right)$.

\section{Ortho-Selective Nitration of Conjugated Styrene Derivatives:}

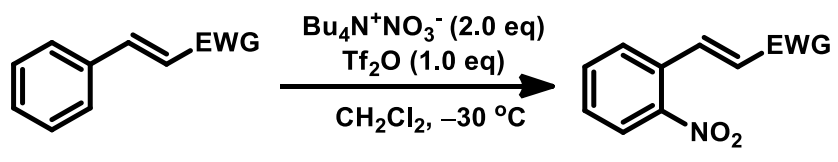

\section{General Procedure for Ortho-Selective Nitration (Method C):}

To a stirred solution of styrene derivative $(3.0 \mathrm{mmol})$ and tetrabutylammonium nitrate $(6.0 \mathrm{mmol})$ in $\mathrm{CH}_{2} \mathrm{Cl}_{2}(5.0 \mathrm{~mL})$ was added trifluoromethanesulfonic anhydride $(3.0 \mathrm{mmol})$ slowly over $30 \mathrm{~min}$. at -30 ${ }^{\circ} \mathrm{C}$. After stirring for $30 \mathrm{~min}$ at $-30{ }^{\circ} \mathrm{C}$, the nitration was complete as monitored by TLC. Then the reaction mixture was treated with solid $\mathrm{NaHCO}_{3}(3.0 \mathrm{mmol})$, filtered and concentrated under reduced pressure. 
The crude product was purified by column chromatography $\left(\mathrm{SiO}_{2}\right.$, hexanes/ethyl acetate) to afford the pure ortho-nitro styrene derivative.

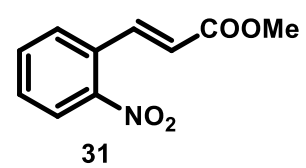

(E)-Methyl 3-(2-nitrophenyl)acrylate (31):

The compound $\mathbf{3 1}{ }^{5}$ (528 mg) was prepared from methyl trans-cinnamate (30) (486 mg, $3.0 \mathrm{mmol}$ ) using method C. (85\% yield) after flash chromatography $\left(\mathrm{SiO}_{2}\right.$, hexanes/ethyl acetate 9:1) and isolated as a white solid. M. P: $71-72{ }^{\circ} \mathrm{C}$; FTIR (neat, $\mathbf{~ c m}^{-1}$ ): 3028, 2952, 1718, 1638, 1571, 1438, 1345, 975; ${ }^{1} \mathbf{H}$ NMR (CDCl 3,400 MHz): $\delta 8.11(\mathrm{~d}, J=15.8 \mathrm{~Hz}, 1 \mathrm{H}), 8.03(\mathrm{~d}, J=7.9 \mathrm{~Hz}, 1 \mathrm{H}), 7.68-7.62(\mathrm{~m}, 2 \mathrm{H})$, $7.56-7.52(\mathrm{~m}, 1 \mathrm{H}), 6.36(\mathrm{~d}, J=15.8 \mathrm{~Hz}, 1 \mathrm{H}), 3.82(\mathrm{~s}, 3 \mathrm{H}) ;{ }^{13} \mathbf{C} \mathbf{N M R}\left(\mathbf{C D C l}_{3}, \mathbf{1 0 0} \mathbf{M H z}\right): \delta 166.2$, 148.3, 140.1 133.5, 130.5, 130.3, 129.1, 124.9, 122.9, 52.0; HRMS (ESI) m/z: $[\mathrm{M}+\mathrm{H}]^{+}$Calcd for $\mathrm{C}_{10} \mathrm{H}_{10} \mathrm{NO}_{4}$ 208.0604; Found 208.0608.

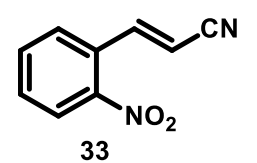

\section{(E)-3-(2-Nitrophenyl)acrylonitrile (33):}

The compound $\mathbf{3 3}^{6}$ (429 mg) was prepared from cinnamonitrile (32) (387 mg, $3.0 \mathrm{mmol}$ ) using method C. (82\% yield) after flash chromatography $\left(\mathrm{SiO}_{2}\right.$, hexanes/ethyl acetate 3:1) and isolated as a colorless crystalline solid. M. P: $87-88^{\circ}$ C; FTIR (neat, $\mathbf{c m}^{-1}$ ): 3102, 3075, 2224, 1602, 1567, 1518, 1441, 1341, 957; ${ }^{1} \mathbf{H}$ NMR $\left(\mathbf{C D C l}_{3}, \mathbf{4 0 0} \mathbf{M H z}\right): \delta 8.13(\mathrm{~d}, J=8.1 \mathrm{~Hz}, 1 \mathrm{H}), 7.97(\mathrm{~d}, J=16.5 \mathrm{~Hz}, 1 \mathrm{H}), 7.71(\mathrm{t}, J=7.6$ $\mathrm{Hz}, 1 \mathrm{H}), 7.62(\mathrm{t}, J=7.8 \mathrm{~Hz}, 1 \mathrm{H}), 7.57(\mathrm{~d}, J=7.8 \mathrm{~Hz}, 1 \mathrm{H}), 5.85(\mathrm{~d}, J=16.4 \mathrm{~Hz}, 1 \mathrm{H}) ;{ }^{13} \mathbf{C ~ N M R}\left(\mathbf{C D C l}_{3}\right.$, 100 MHz): $\delta$ 147.5, 146.6, 134.0, 131.3, 129.7, 128.7, 125.4, 116.9, 101.6; HRMS (ESI) m/z: [M + H] ${ }^{+}$ Calcd for $\mathrm{C}_{9} \mathrm{H}_{7} \mathrm{~N}_{2} \mathrm{O}_{2}$ 175.0502; Found 175.0505.

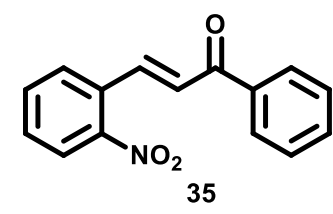

\section{(E)-3-(2-Nitrophenyl)-1-phenylprop-2-en-1-one (35):}

The compound $\mathbf{3 5}^{7}$ (668 mg) was prepared from benzylideneacetophenone (34) (624 mg, $3.0 \mathrm{mmol}$ ) using method C. (88\% yield) after flash chromatography $\left(\mathrm{SiO}_{2}\right.$, hexanes/ethyl acetate 9:1) and isolated as a faint yellow solid. M. P: $111-113{ }^{\circ}$ C; FTIR (neat, $\mathbf{c m}^{-1}$ ): 3063, 2923, 1664, 1604, 1570, 1519, 1445, 1340, 1214, 1012, 740; ${ }^{1} \mathbf{H}$ NMR (CDCl $\mathbf{3}, 400$ MHz): $\delta 8.14(\mathrm{~d}, J=15.7 \mathrm{~Hz}, 1 \mathrm{H}), 8.08(\mathrm{~d}, J=8.1 \mathrm{~Hz}, 1 \mathrm{H}), 8.04$ 
- $8.00(\mathrm{~m}, 2 \mathrm{H}), 7.78-7.72(\mathrm{~m}, 1 \mathrm{H}), 7.72-7.67(\mathrm{~m}, 1 \mathrm{H}), 7.63-7.56(\mathrm{~m}, 2 \mathrm{H}), 7.55-7.49(\mathrm{~m}, 2 \mathrm{H}), 7.32$ $(\mathrm{d}, J=15.7 \mathrm{~Hz}, 1 \mathrm{H}) ;{ }^{13} \mathbf{C} \mathbf{N M R}\left(\mathbf{C D C l}_{3}, \mathbf{1 0 0} \mathbf{M H z}\right): \delta 190.5,148.6,140.2,137.2,133.6,133.2,131.4$, 130.4, 129.3, 128.8, 128.7, 127.4, 125.0; HRMS (ESI) m/z: $[\mathrm{M}+\mathrm{H}]^{+}$Calcd for $\mathrm{C}_{15} \mathrm{H}_{12} \mathrm{NO}_{3} 254.0812$; Found 254.0815.

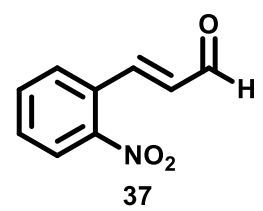

(E)-3-(2-Nitrophenyl)acrylaldehyde (37):

The compound $37^{8}$ (447 mg) was prepared from trans-cinnamaldehyde (36) (396 mg, $3.0 \mathrm{mmol}$ ) using method C. (84\% yield) after flash chromatography $\left(\mathrm{SiO}_{2}\right.$, hexanes/ethyl acetate 9:1) and isolated as a white crystalline solid. M. P: $97-98{ }^{\circ}$ C; FTIR (neat, $\mathbf{~ c m}^{-1}$ ): 3061, 2929, 1666, 1600, 1565, 1515, 1447, 1337, 1220, 1013, 743; ${ }^{1} \mathbf{H}$ NMR (CDCl 3 , 400 MHz): $\delta 9.79(\mathrm{~d}, J=7.6 \mathrm{~Hz}, 1 \mathrm{H}), 8.11$ (dd, $J=8.1,1.2$ $\mathrm{Hz}, 1 \mathrm{H}), 8.04(\mathrm{~d}, J=15.8 \mathrm{~Hz}, 1 \mathrm{H}), 7.74-7.67(\mathrm{~m}, 2 \mathrm{H}), 7.64-7.58(\mathrm{~m}, 1 \mathrm{H}), 6.64(\mathrm{dd}, J=15.8,7.7 \mathrm{~Hz}$, 1H); ${ }^{13} \mathbf{C}$ NMR $\left(\mathbf{C D C l}_{3}, \mathbf{1 0 0} \mathbf{M H z}\right): \delta 193.1,148.1,147.3,133.8,132.7,131.1,130.0,129.1,125.2$; HRMS (ESI) m/z: [M+ H $]^{+}$Calcd for $\mathrm{C}_{9} \mathrm{H}_{8} \mathrm{NO}_{3}$ 178.0499; Found 178.0501.

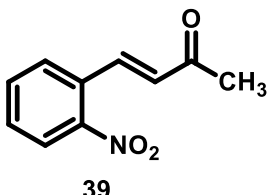

(E)-4-(2-Nitrophenyl)but-3-en-2-one (39):

The compound $3^{9}$ (470 mg) was prepared from benzalacetone (38) (438 mg, $3.0 \mathrm{mmol}$ ) using method C. (82\% yield) after flash chromatography $\left(\mathrm{SiO}_{2}\right.$, hexanes/ethyl acetate 7:3) and isolated as a white solid. $\mathbf{M}$. P: $60-61{ }^{\circ} \mathrm{C}$; FTIR (neat, $\mathbf{c m}^{-1}$ ): 3065, 2839, 1679, 1601, 1516, 1337, 1220, 1121, 979; ${ }^{1} \mathbf{H}$ NMR $\left(\mathbf{C D C l}_{3}, 400 \mathrm{MHz}\right): \delta 8.05(\mathrm{~d}, J=8.7 \mathrm{~Hz}, 1 \mathrm{H}), 7.96(\mathrm{~d}, J=16.2 \mathrm{~Hz}, 1 \mathrm{H}), 7.69-7.62(\mathrm{~m}, 2 \mathrm{H}), 7.55$ (ddd, $J=8.7,6.6,2.3 \mathrm{~Hz}, 1 \mathrm{H}), 6.56(\mathrm{~d}, J=16.2 \mathrm{~Hz}, 1 \mathrm{H}), 2.41(\mathrm{~s}, 3 \mathrm{H}) ;{ }^{13} \mathbf{C} \mathbf{N M R}\left(\mathbf{C D C l}_{3}, \mathbf{1 0 0} \mathbf{M H z}\right): \delta 198.0$, 148.3, 138.9, 133.7, 131.9, 130.7, 130.4, 129.1, 125.0, 27.1; HRMS (ESI) m/z: $[\mathrm{M}+\mathrm{H}]^{+}$Calcd for $\mathrm{C}_{10} \mathrm{H}_{10} \mathrm{NO}_{3}$ 192.0655; Found 192.0659.

\section{Para- Selective Nitration:}

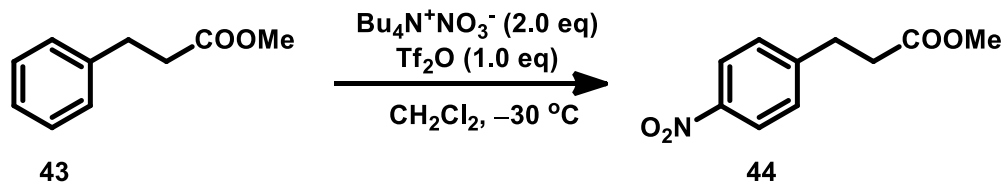




\section{Methyl 3-(4-nitrophenyl)propanoate (44):}

The compound $\mathbf{4 4}^{10}$ (539 mg) was prepared from methyl 3-phenylpropionate (43) (492 mg, $3.0 \mathrm{mmol}$ ) using method C. (86\% yield) after flash chromatography $\left(\mathrm{SiO}_{2}\right.$, hexanes/ethyl acetate 10:1) and isolated as a faint yellow crystalline solid. M. P: $68-70{ }^{\circ}$ C; FTIR (neat, $\mathbf{~ c m}^{-1}$ ): 3081, 2954, 1728, 1637, 1599, 1517, 1426, 1168, 854; ${ }^{1} \mathbf{H}$ NMR ( $\mathbf{C D C l}_{3}, 400$ MHz): $\delta 8.16$ - $8.10(\mathrm{~m}, 2 \mathrm{H}), 7.38-7.34$ (m, 2H), 3.66 (s, $3 \mathrm{H}), 3.05(\mathrm{t}, J=7.5 \mathrm{~Hz}, 2 \mathrm{H}), 2.67(\mathrm{t}, J=7.5 \mathrm{~Hz}, 2 \mathrm{H}) ;{ }^{13} \mathbf{C} \mathbf{N M R}\left(\mathbf{C D C l}_{3}, \mathbf{1 0 0} \mathbf{M H z}\right): \delta 172.6,148.2$, 146.7, 129.2, 123.8, 51.8, 34.8, 30.6; HRMS (ESI) m/z: $[\mathrm{M}+\mathrm{H}]^{+}$Calcd for $\mathrm{C}_{10} \mathrm{H}_{12} \mathrm{NO}_{4}$ 232.0580; Found 232.0570 .

\section{References:}

1) (a) Reddy, G. S.; Reddy, D. S.; Corey, E. J. Unraveling the $\mathrm{C}_{2}$ - Symmetric Azatetraquinane System. Simple, Enantioselective Syntheses. Org. Lett. 2021, 23, 2258-2262. (b) Huw, J. R.; Chen, K. L.; Ananthan, S.; Patel, H. V. Ultrasonic Nitration of Allylsilanes by Use of Sodium Nitrite and Ceric Ammonium Nitrate. Organometallics. 1996, 15, 499-505.

2) Corey, E. J.; Estreicher, H. Nitrodestannylation. A New Synthesis of Conjugated Nitro Cyclo Olefins. Tetrahedron Lett. 1980, 21, 1113-1116.

3) Kim, W. H.; Lee, J. H.; Aussedat, B.; Danishefsky, S. J. A Diels-Alder route to angularly functionalized bicyclic structures. Tetrahedron. 2010, 66, 6391-6398.

4) Kancharla, P. K.; Reddy, Y. S.; Dharuman, S.; Vankar, Y. D. Acetyl Chloride-Silver NitrateAcetonitrile: A Reagent System for the Synthesis of 2-Nitroglycals and 2-Nitro-1-Acetamido Sugars from Glycals. J. Org. Chem. 2011, 76, 5832-5837.

5) Le Callonnec, F.; Fouquet, E.; Felpin, F.-X. Unprecedented Substoichiometric Use of Hazardous Aryl Diazonium Salts in the Heck-Matsuda Reaction via a Double Catalytic Cycle. Org. Lett. 2011, 13, 2646-2649.

6) Wu, Q.; Luo, Y.; Lei, A.; You, J. Aerobic Copper-promoted radical-type cleavage of coordinated cyanide anion: Nitrogen transfer to aldehydes to form nitriles. J. Am. Chem. Soc. 2016, 138, 2885-2888.

7) Jiang, Q.; Jia, J.; Xu, B.; Zhao, A.; Guo, C. C. Iron-Facilitated Oxidative Radical Decarboxylative Cross-Coupling between $\alpha$-Oxocarboxylic Acids and Acrylic Acids: An Approach to $\alpha, \beta$ Unsaturated Carbonyls. J. Org. Chem. 2015, 80, 3586-3596.

8) Hayashi, Y.; Sakamoto, D.; Okamura, D. One-Pot Synthesis of (S)-Baclofen via Aldol Condensation of Acetaldehyde with Diphenylprolinol Silyl Ether Mediated Asymmetric Michael Reaction as a Key Step. Org. Lett. 2016, 18, 4-7. 
9) Feng, X. W.; Li, C.; Wang, N.; Li, K.; Zhang, W. W.; Wang, Z.; Yu, X. Q. Lipase-catalysed decarboxylative aldol reaction and decarboxylative Knoevenagel reaction. Green Chem. 2009, 11, 1933-1936.

10) Shibata, I.; Suwa, T.; Ryu, K.; Baba, A. Conjugate Hydrostannation of Unsaturated Esters by Iodotin Hydride Ate Complex. J. Org. Chem. 2001, 66, 8690-8692. 


\section{Copies of ${ }^{1} \mathrm{H}$ and ${ }^{13} \mathrm{C}$ NMR Spectra:}
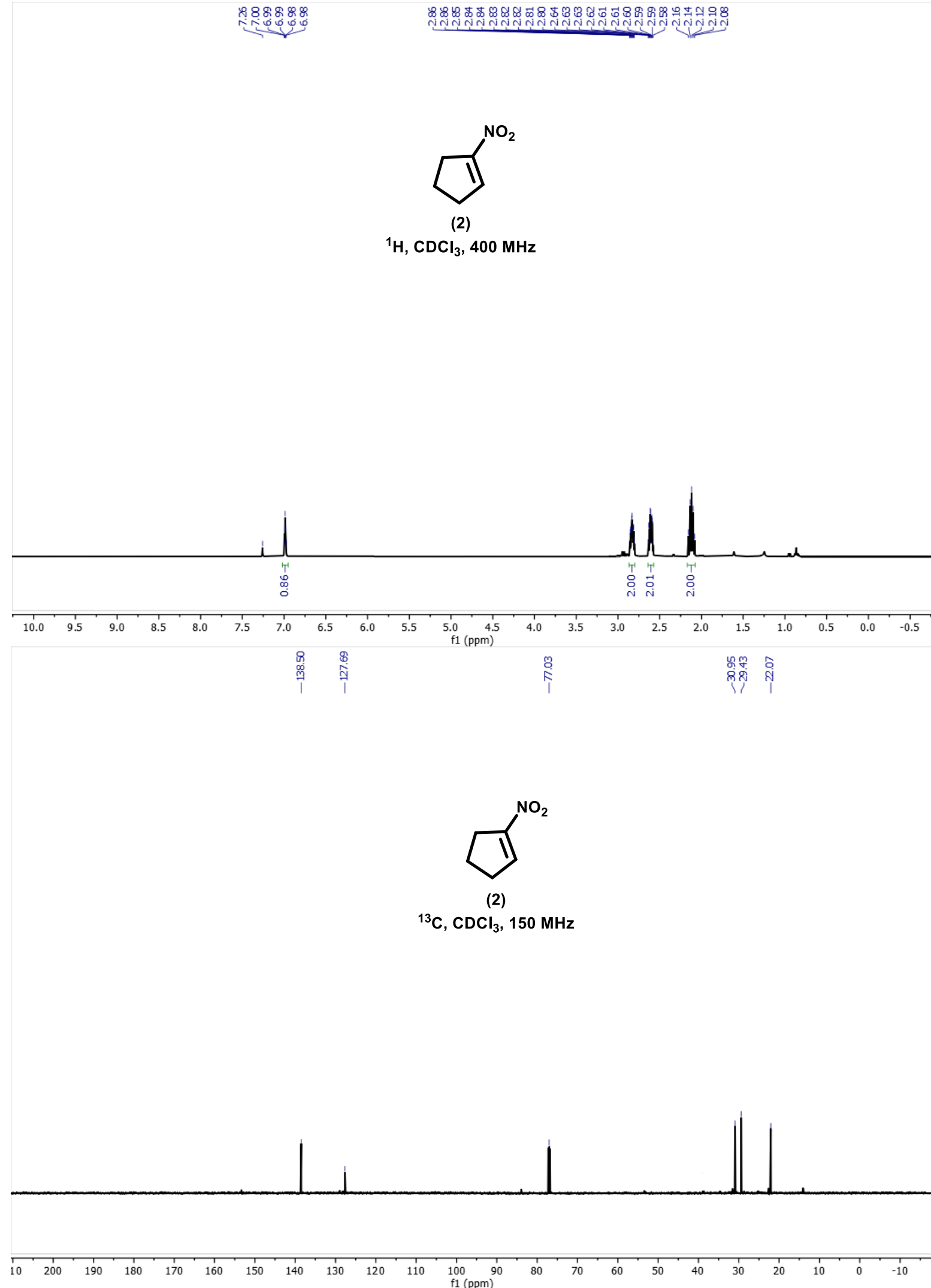


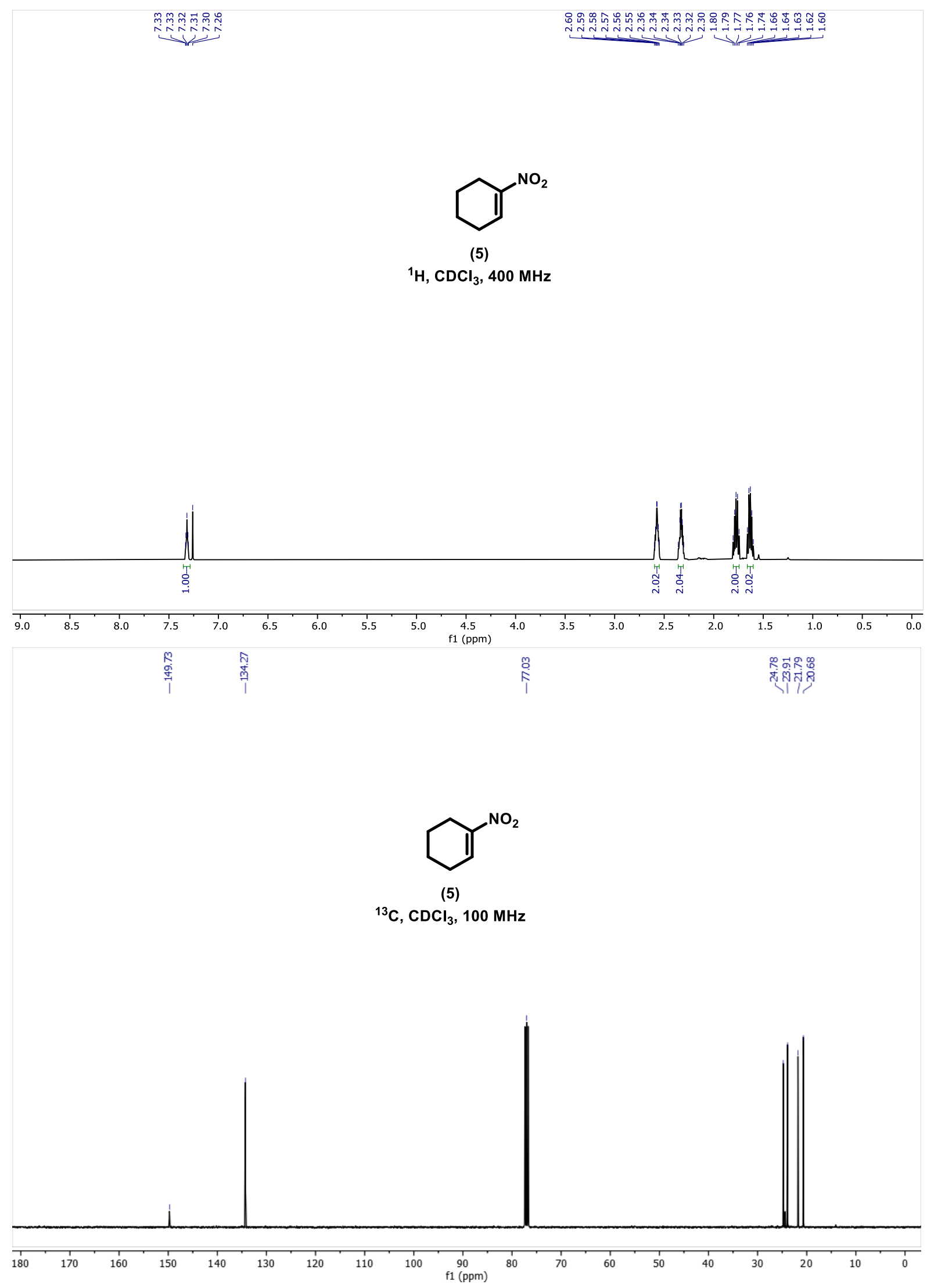




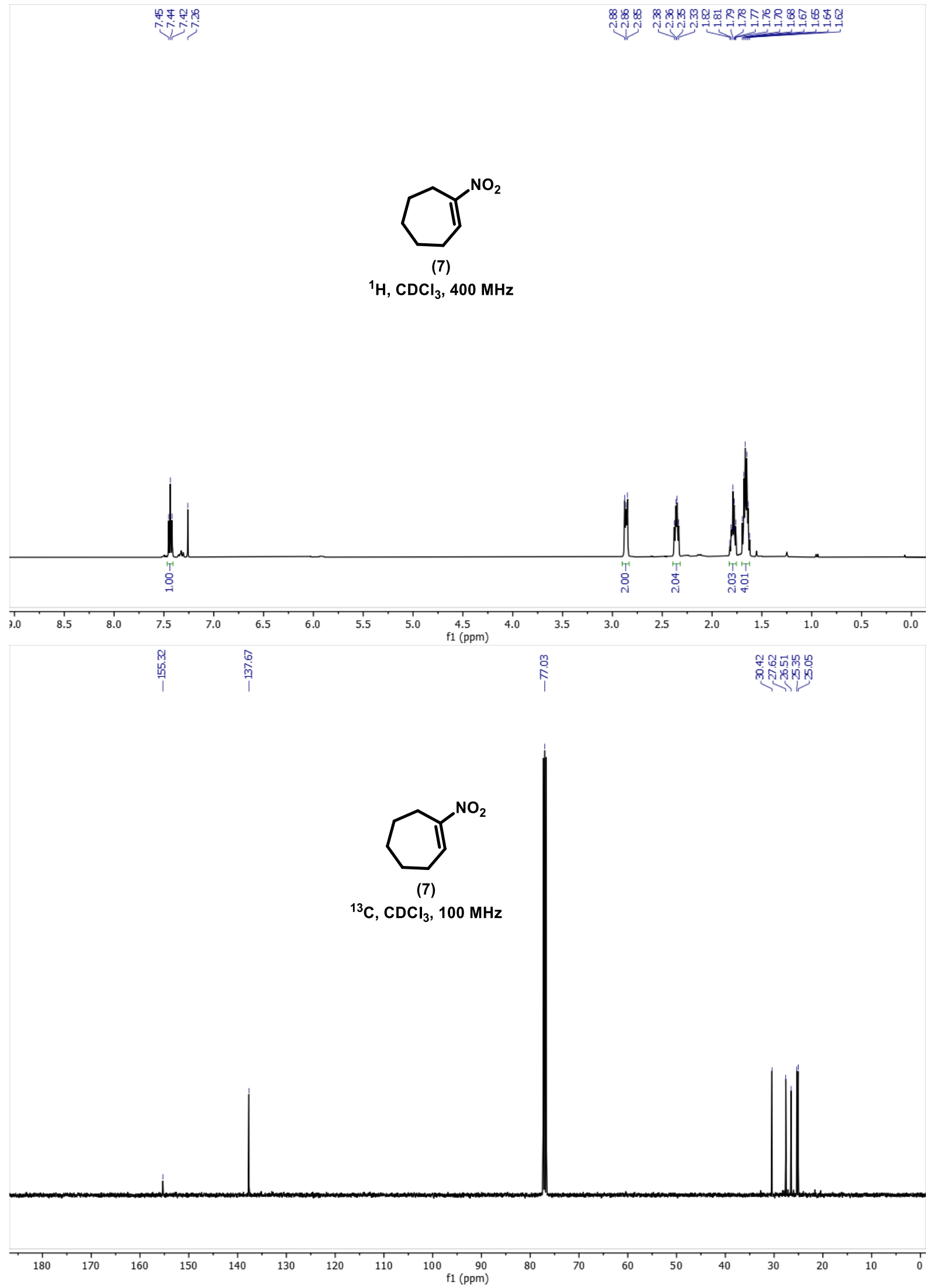




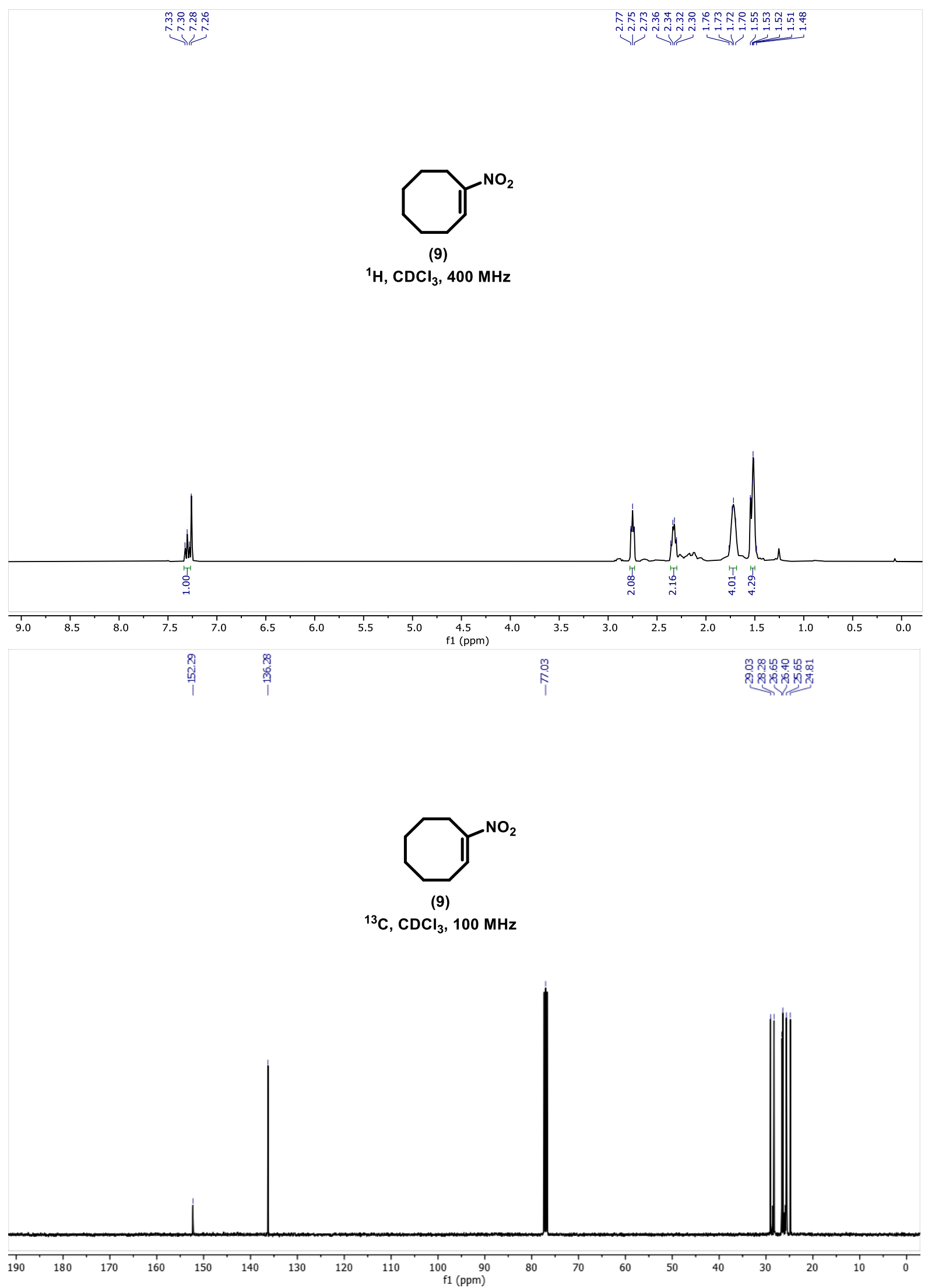




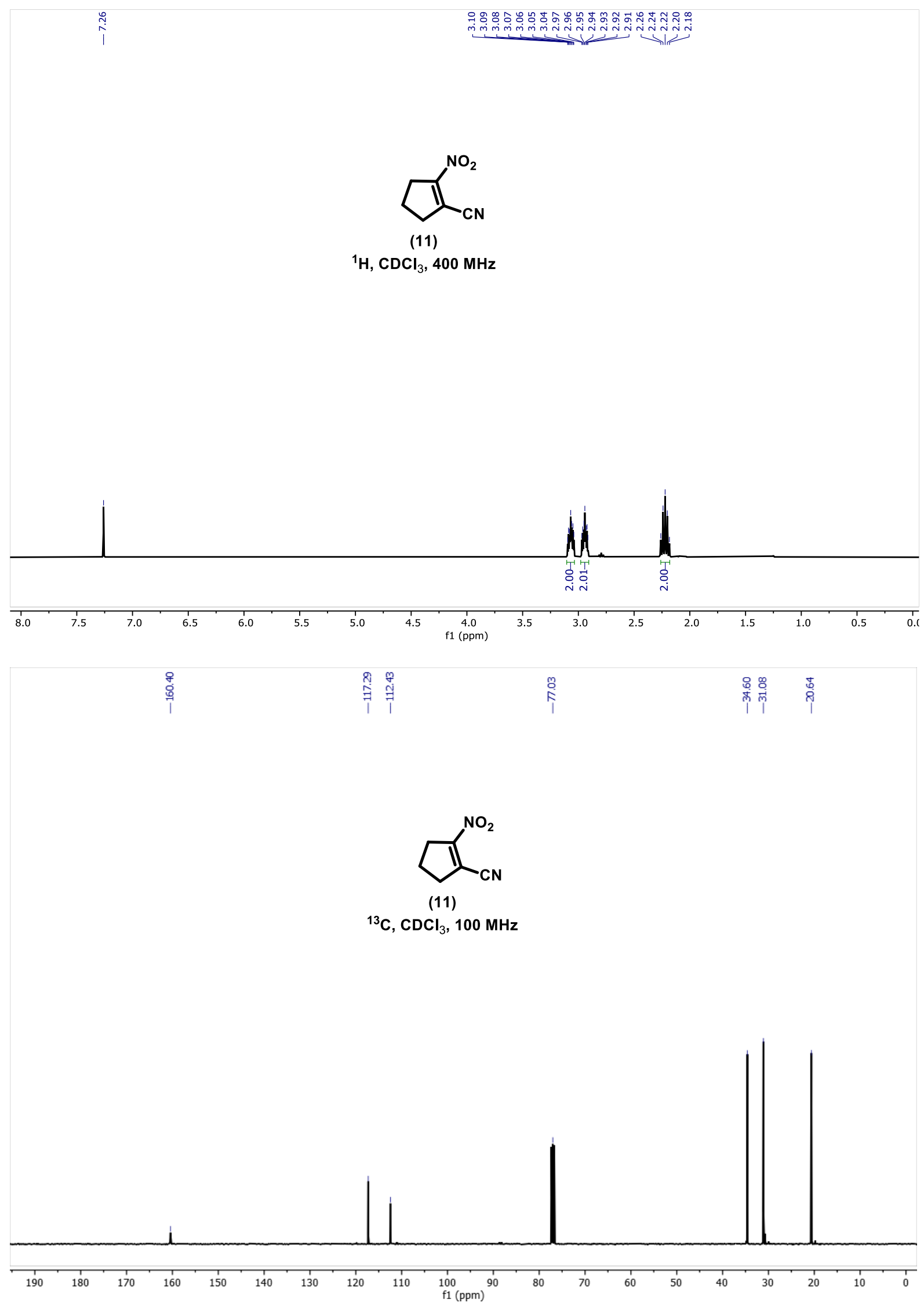




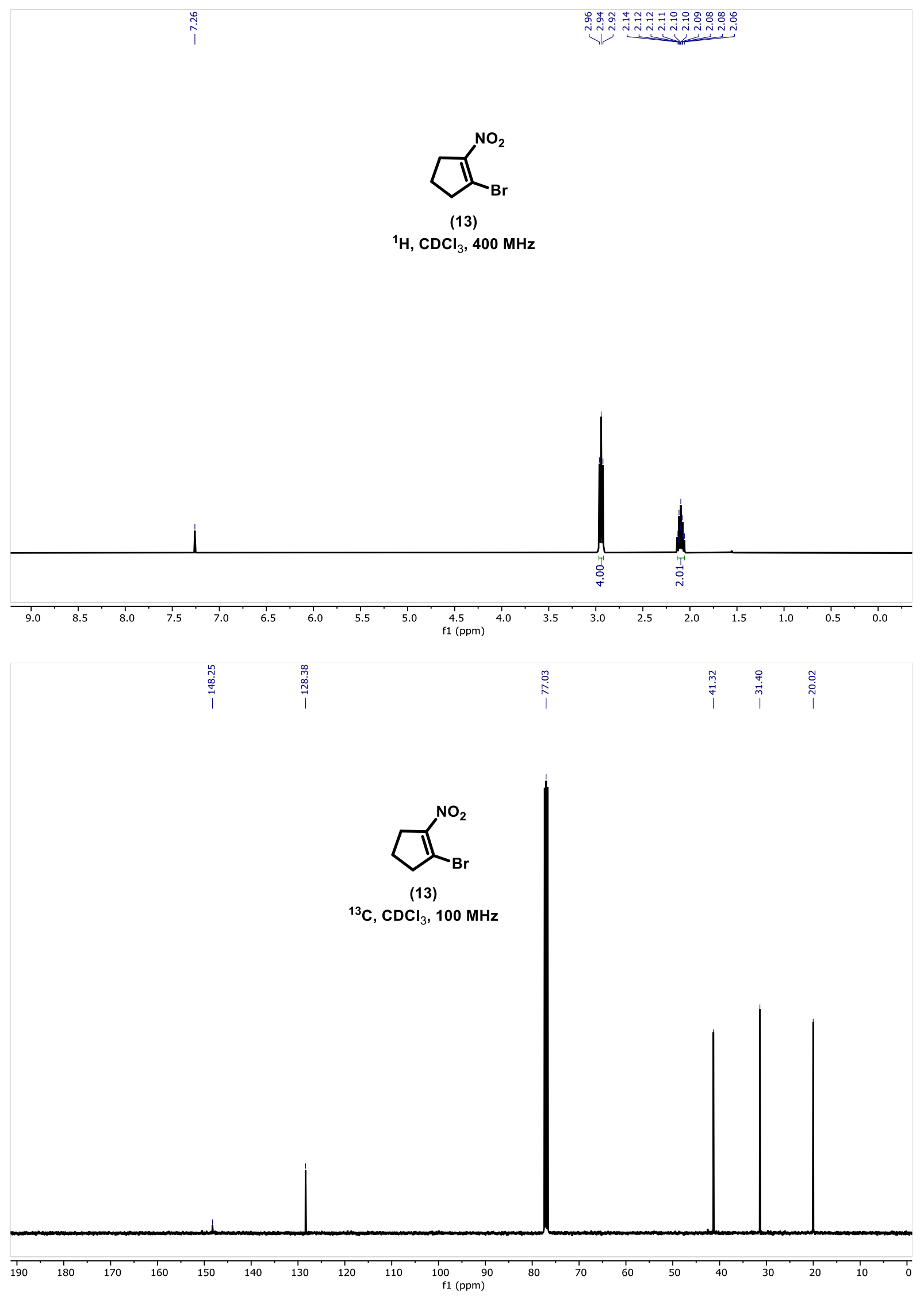




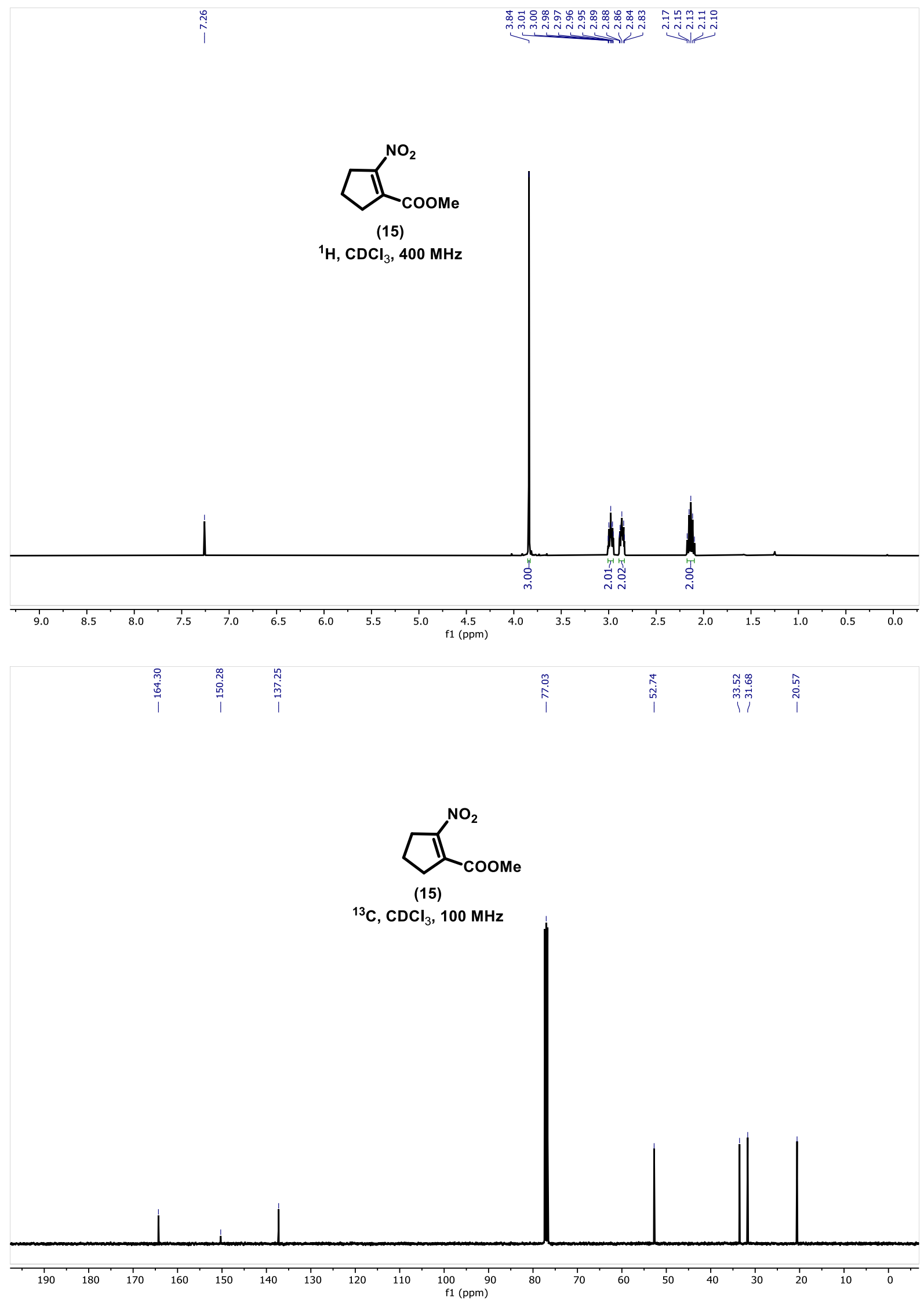




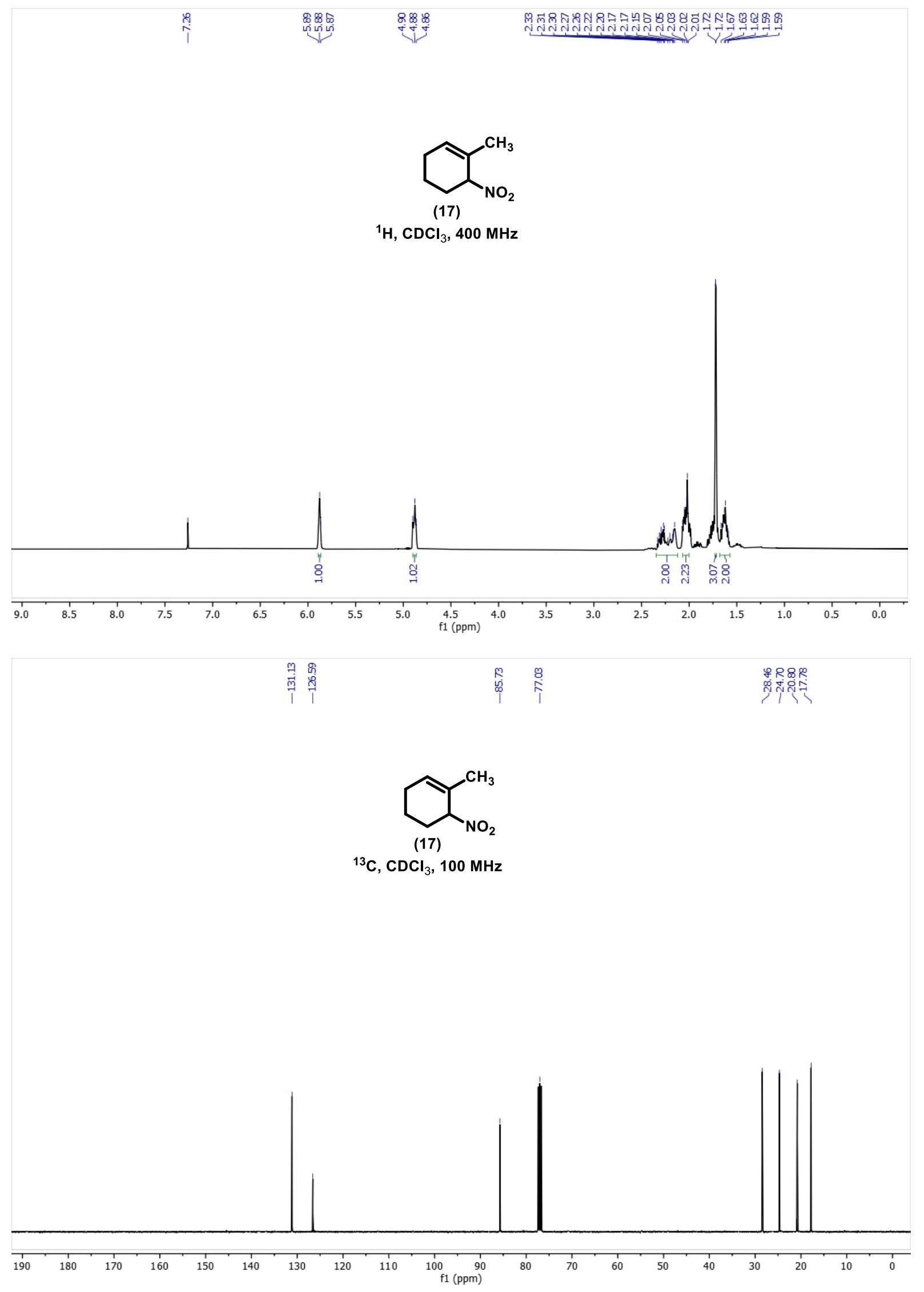




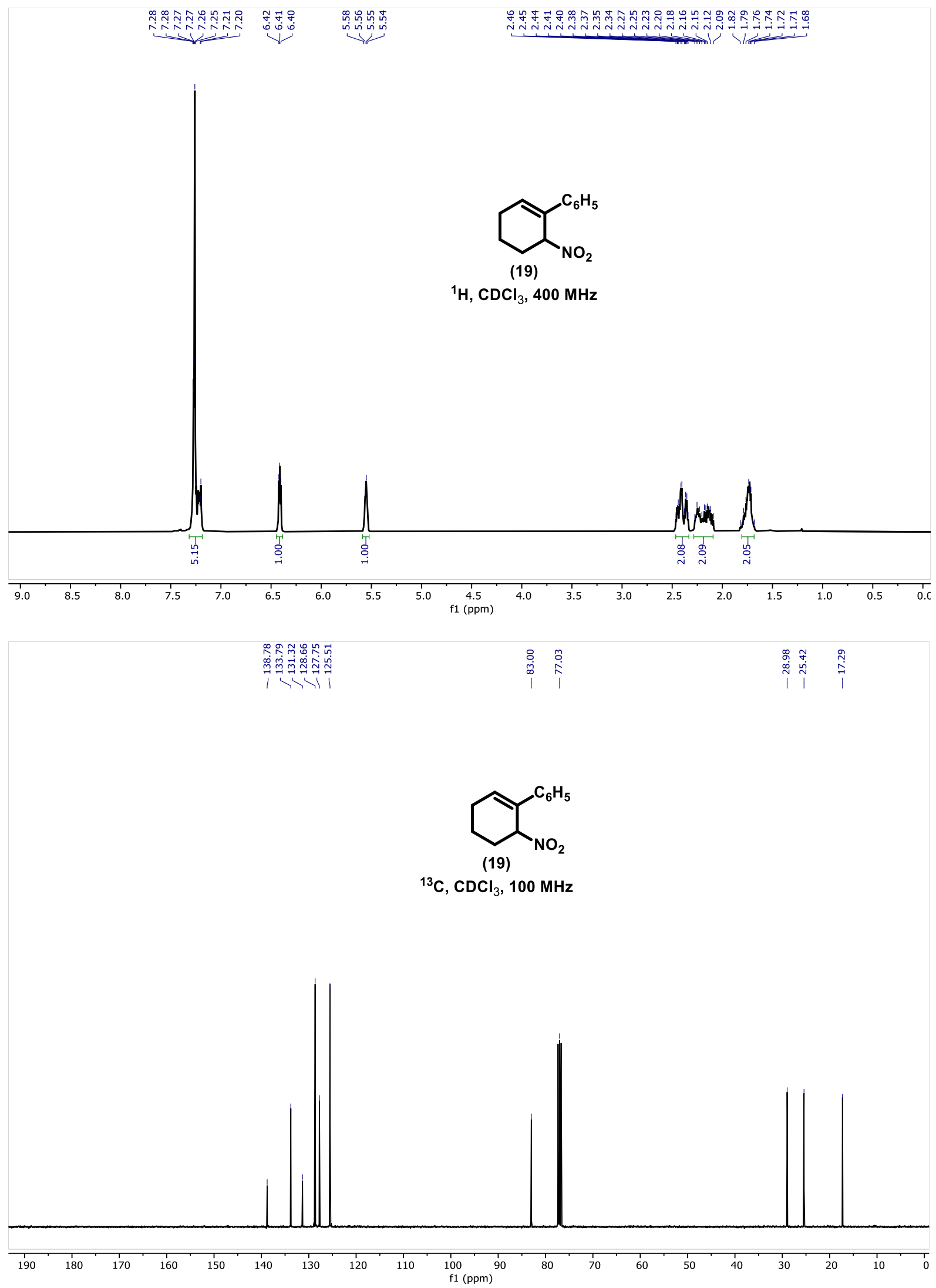




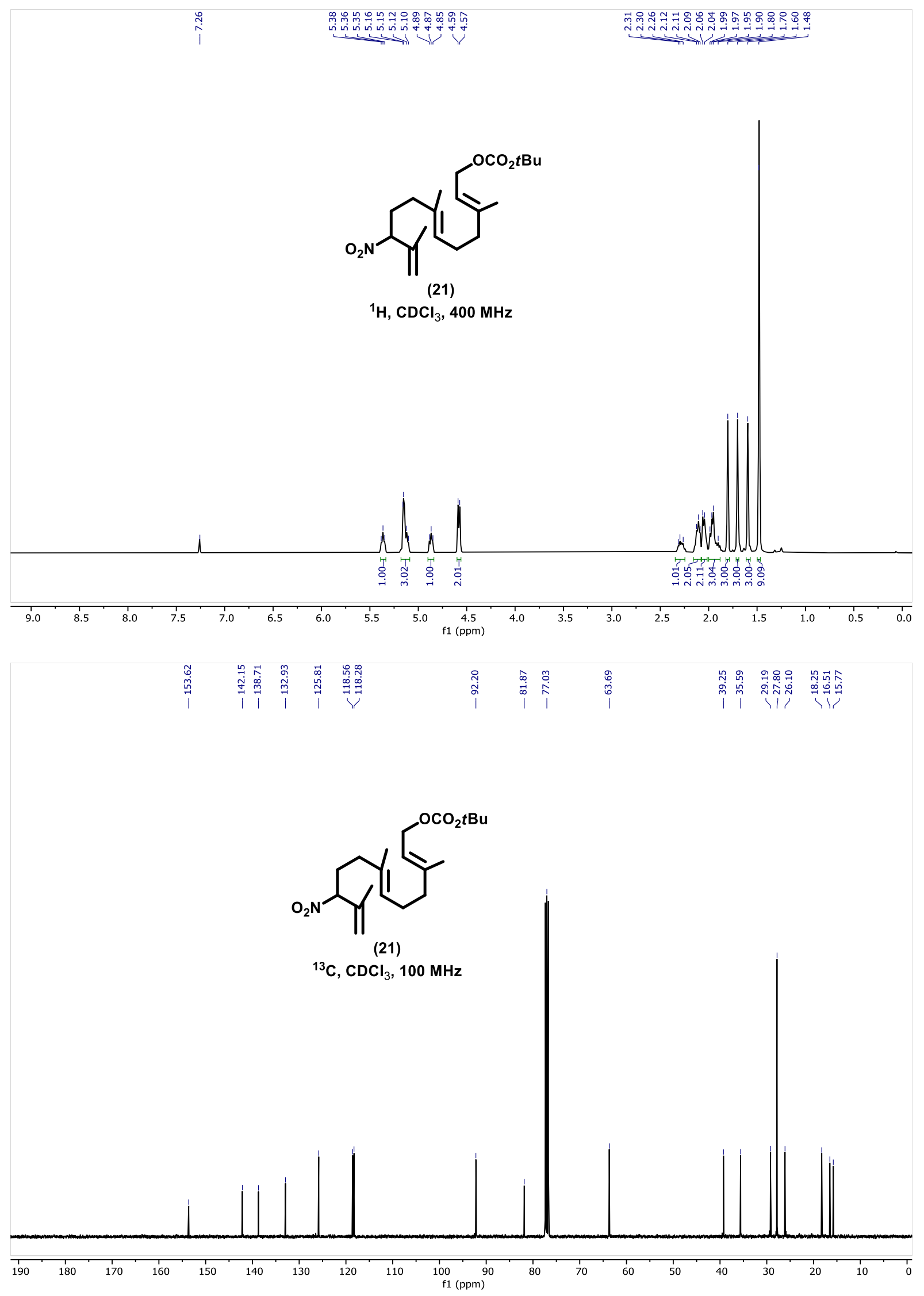




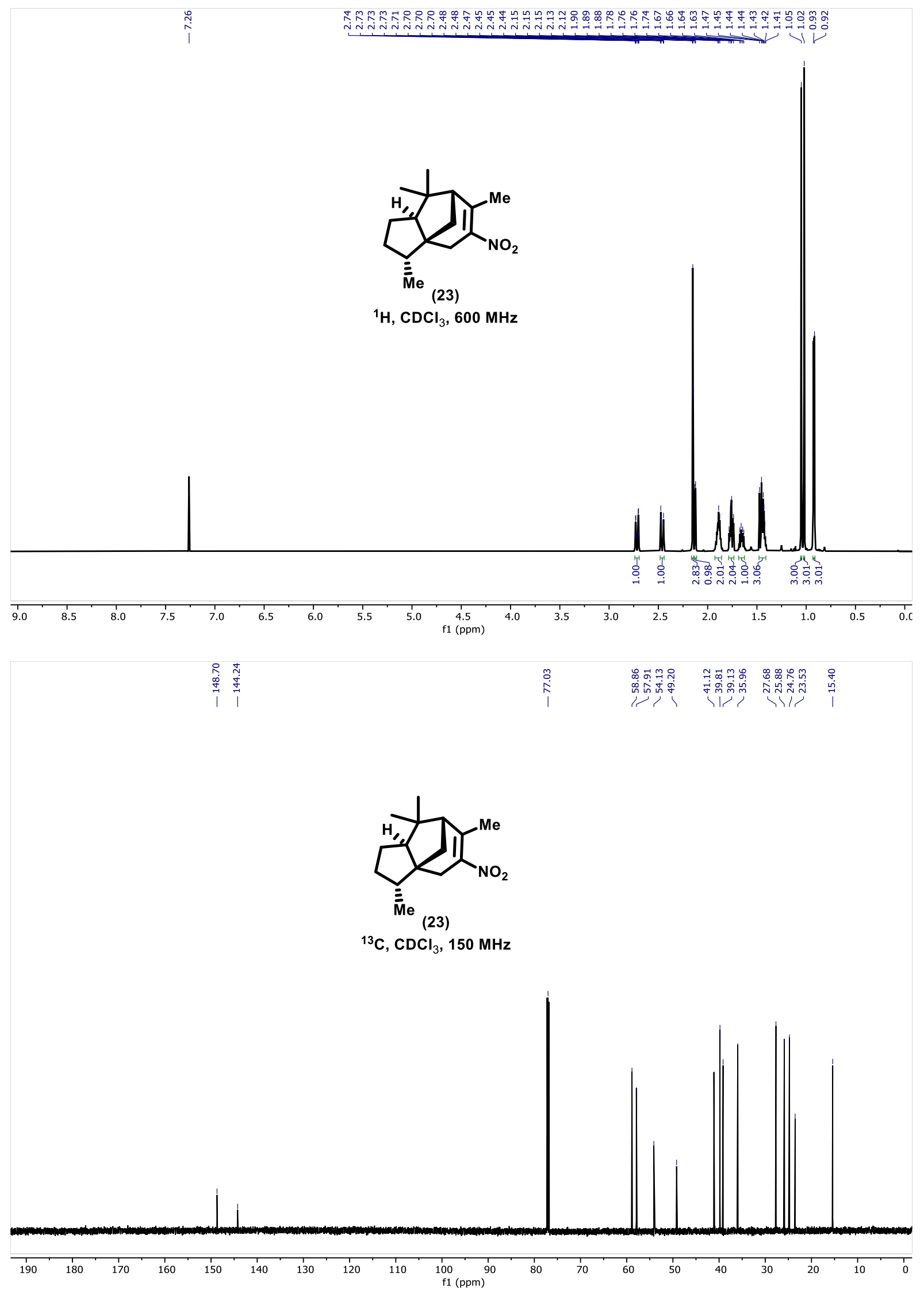




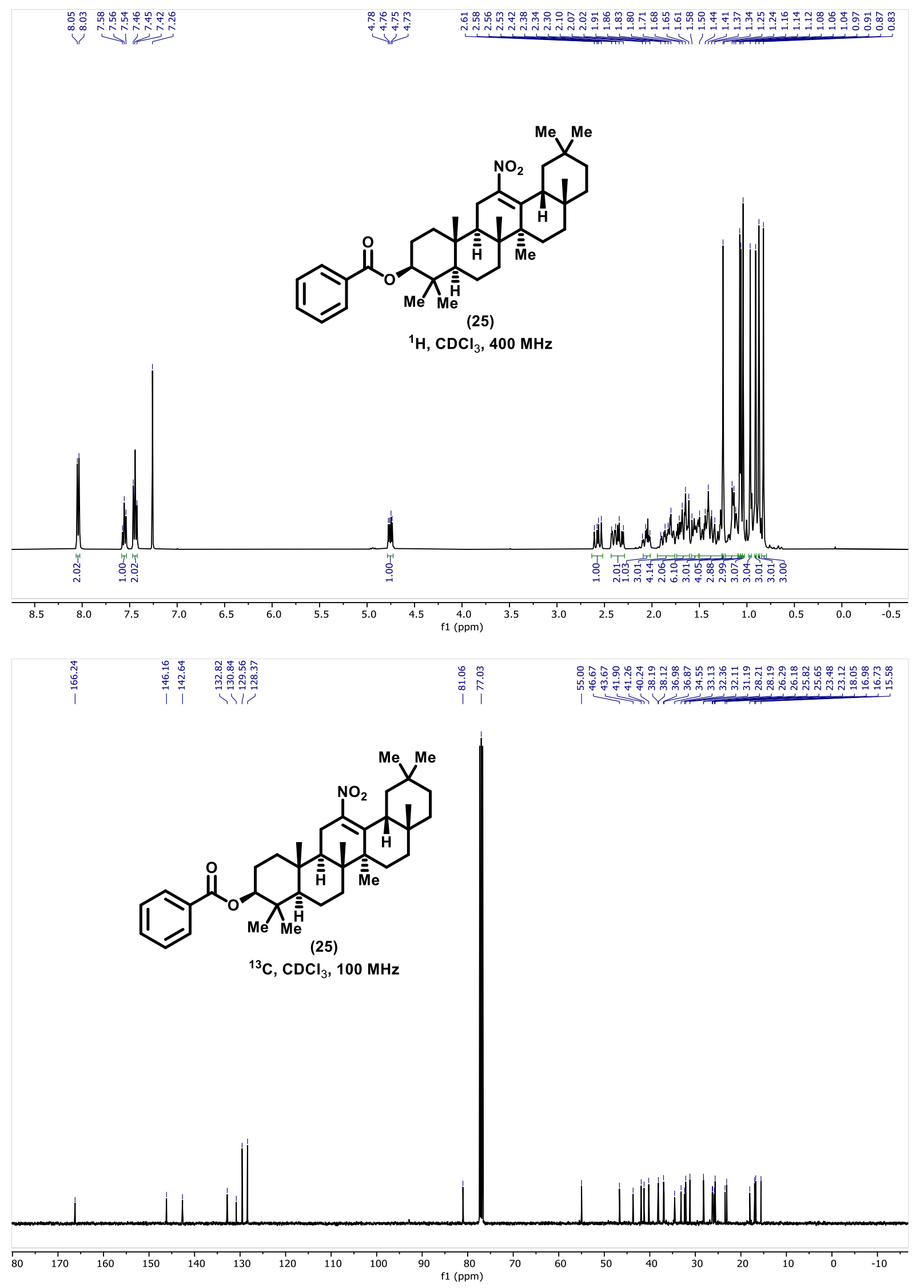



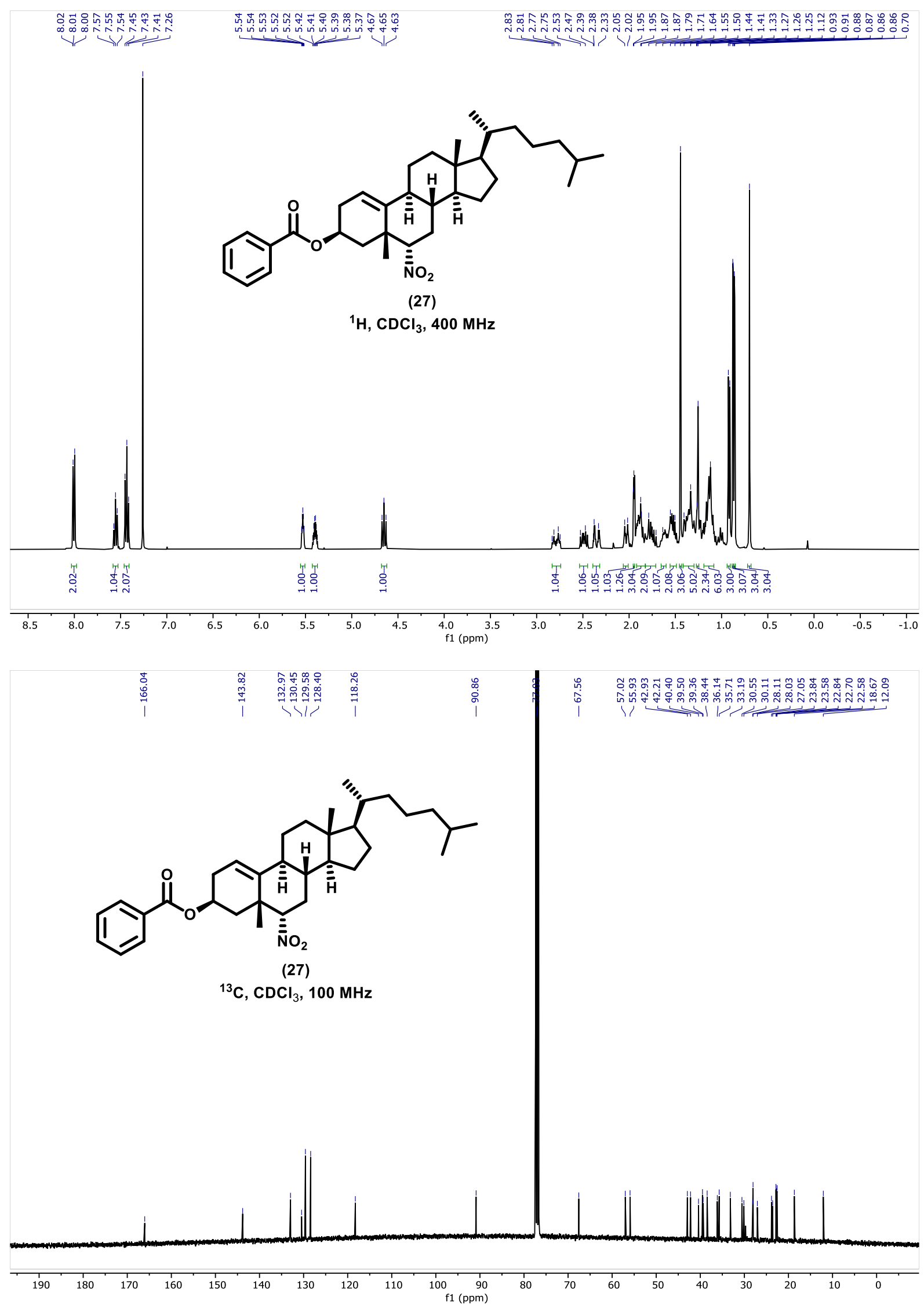

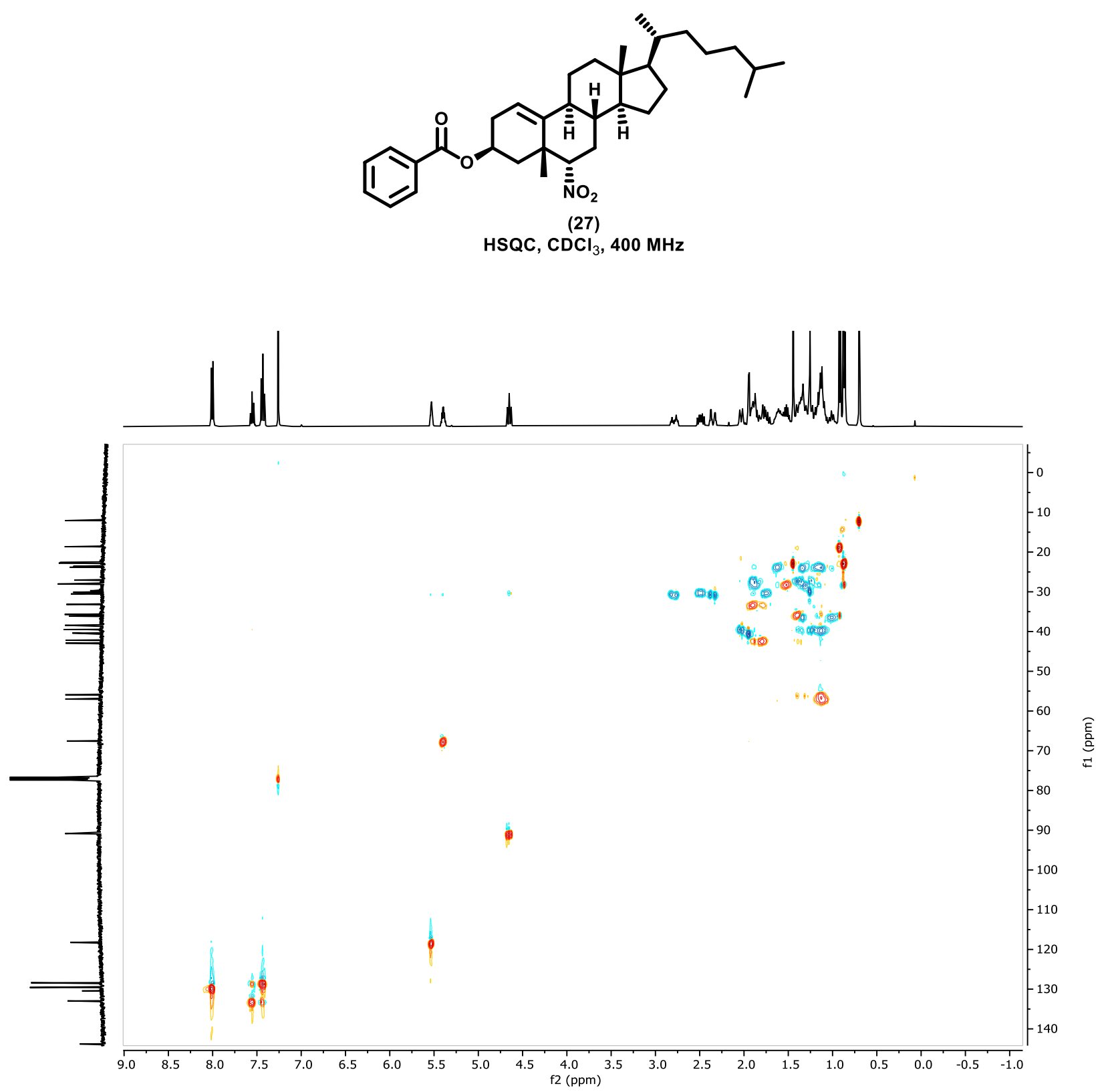


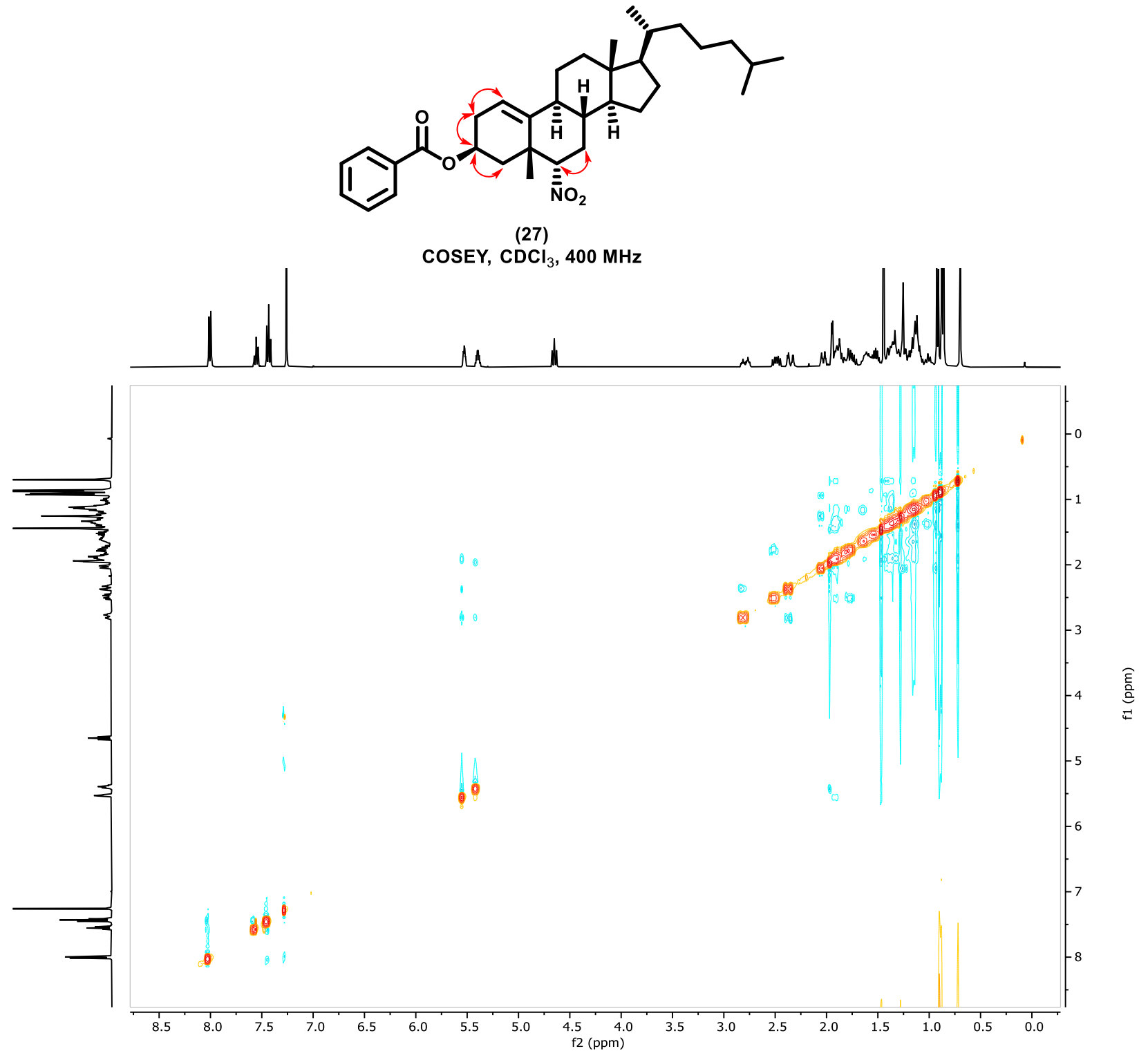



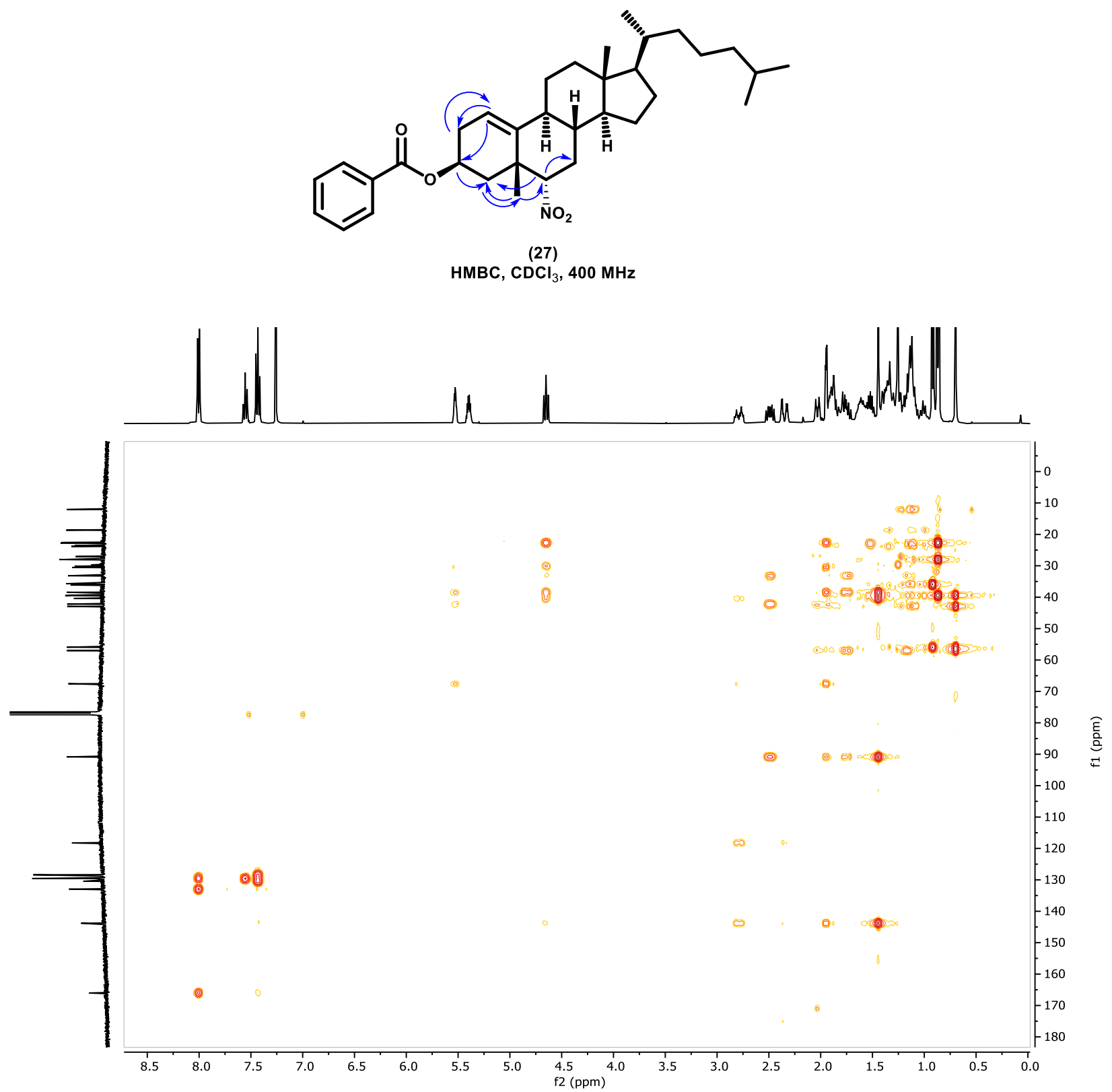


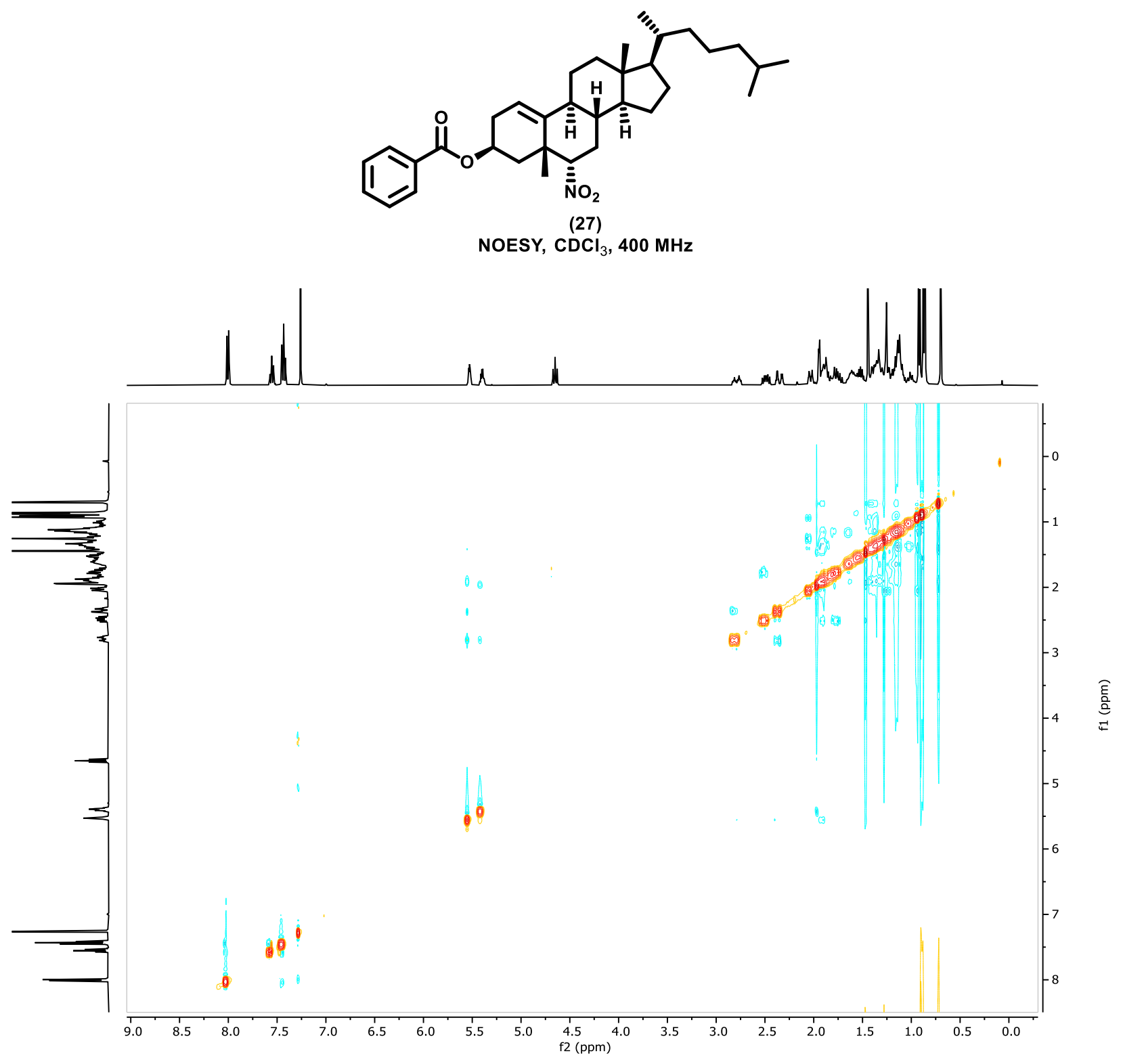



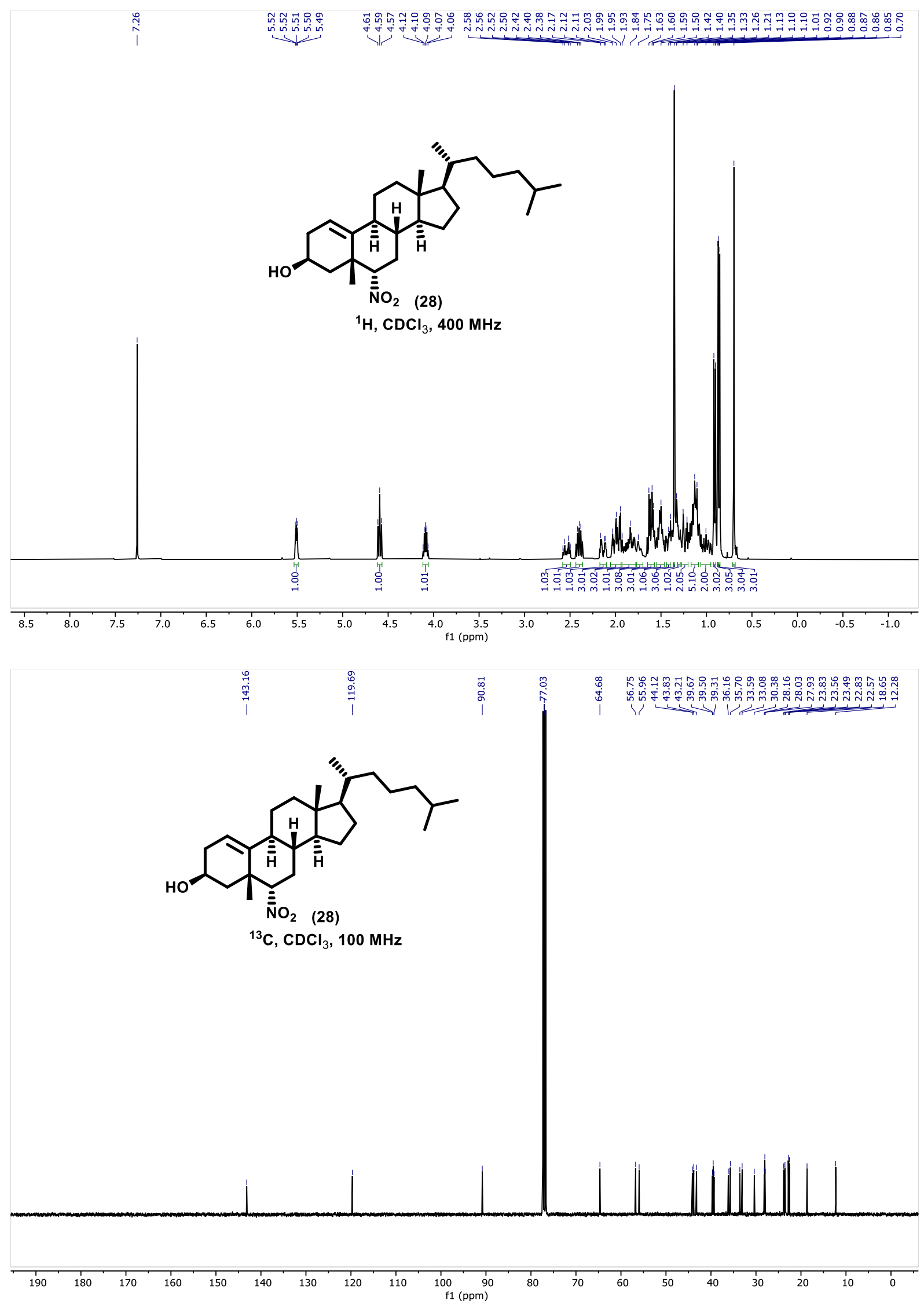


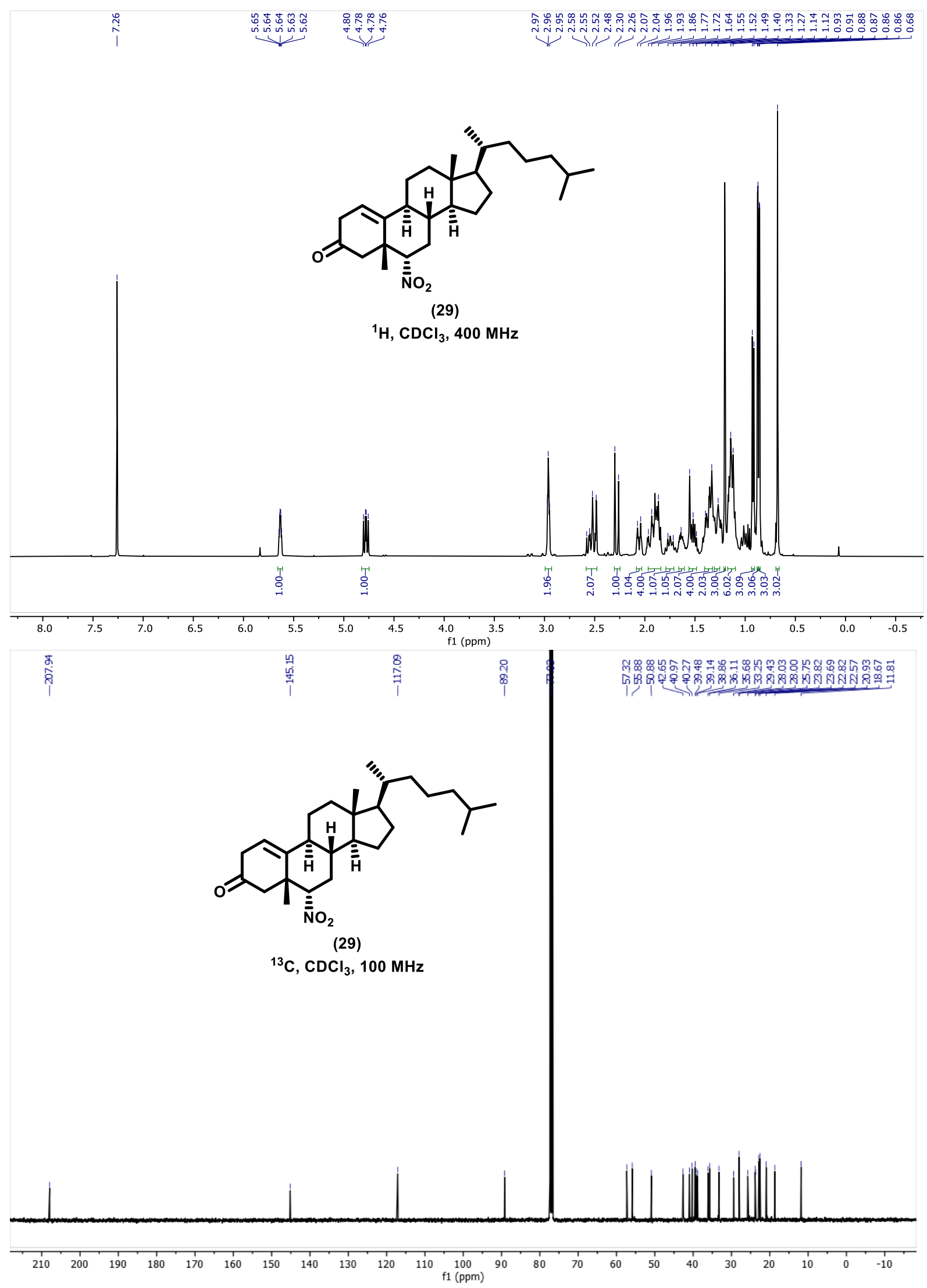




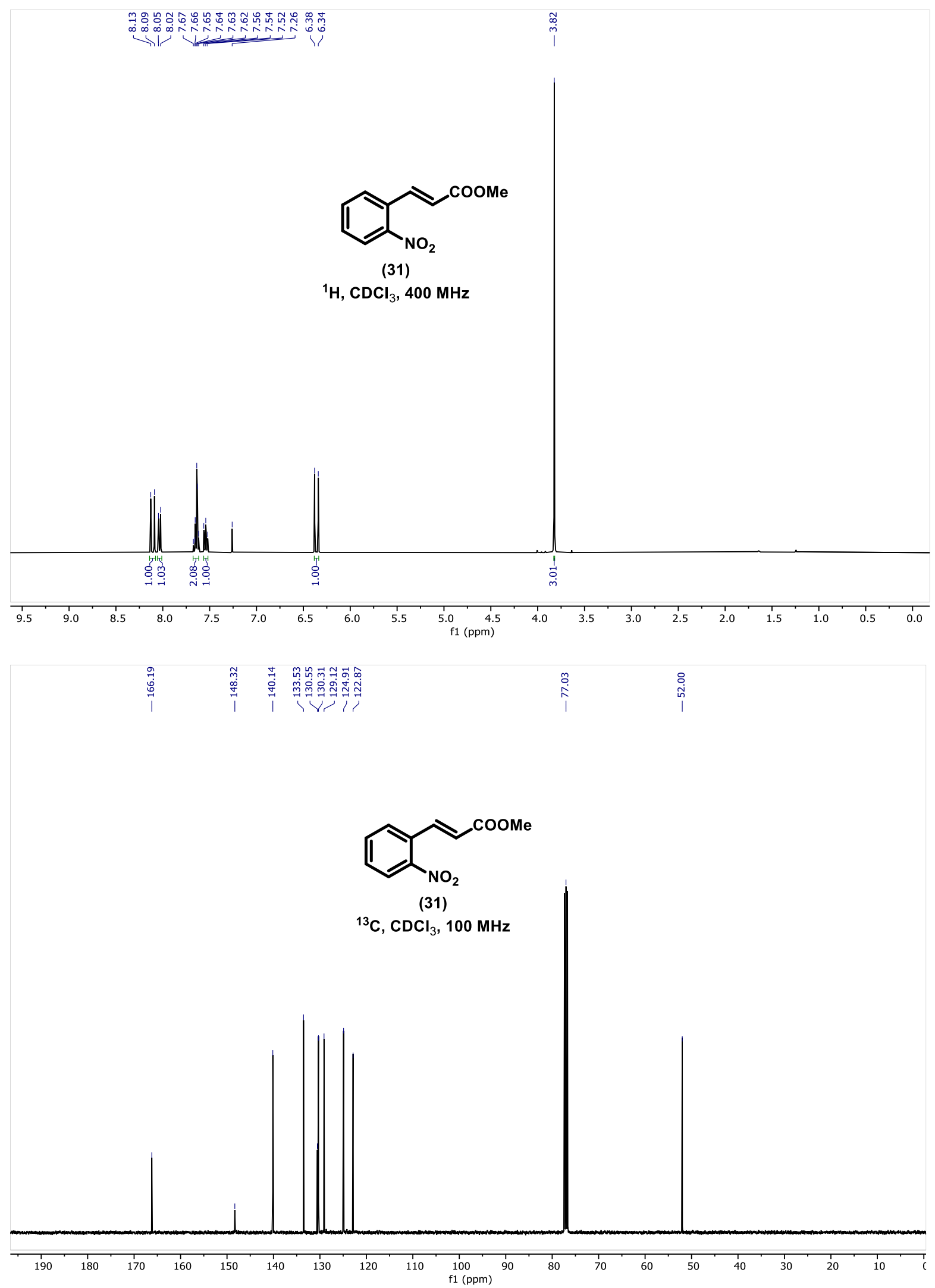




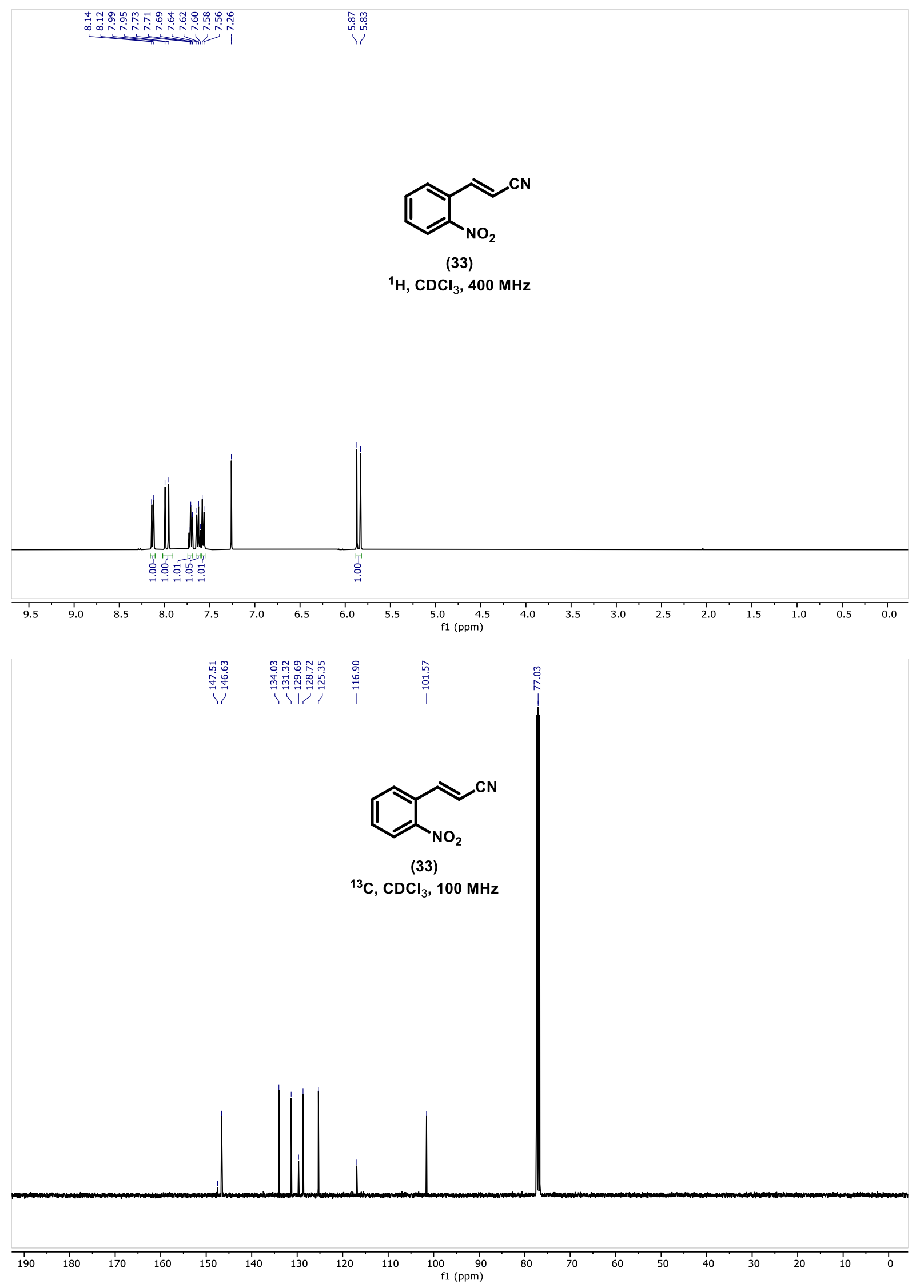




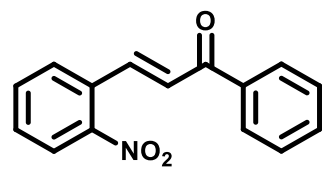

(35)

${ }^{1} \mathrm{H}, \mathrm{CDCl}_{3}, 400 \mathrm{MHz}$
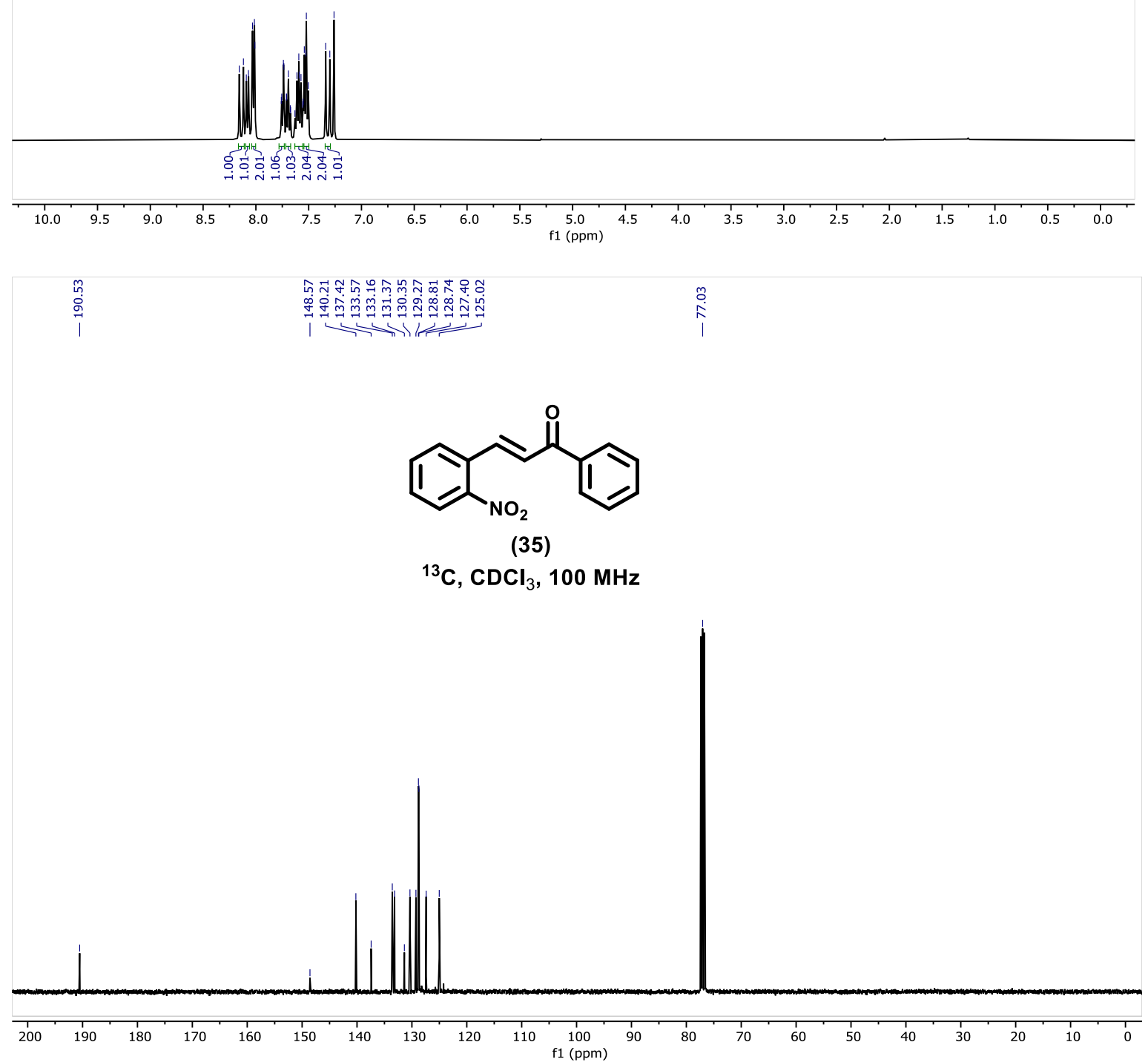


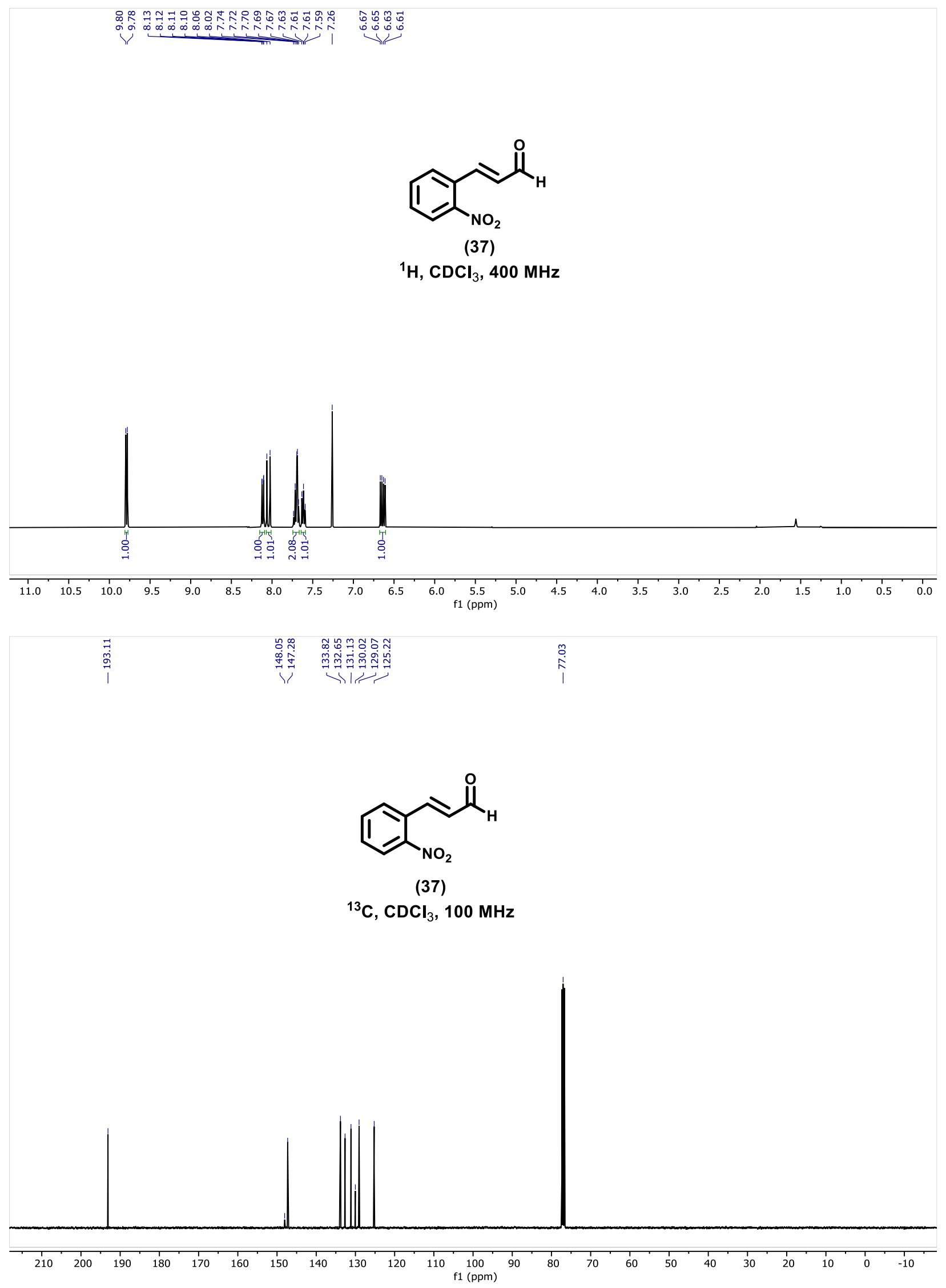




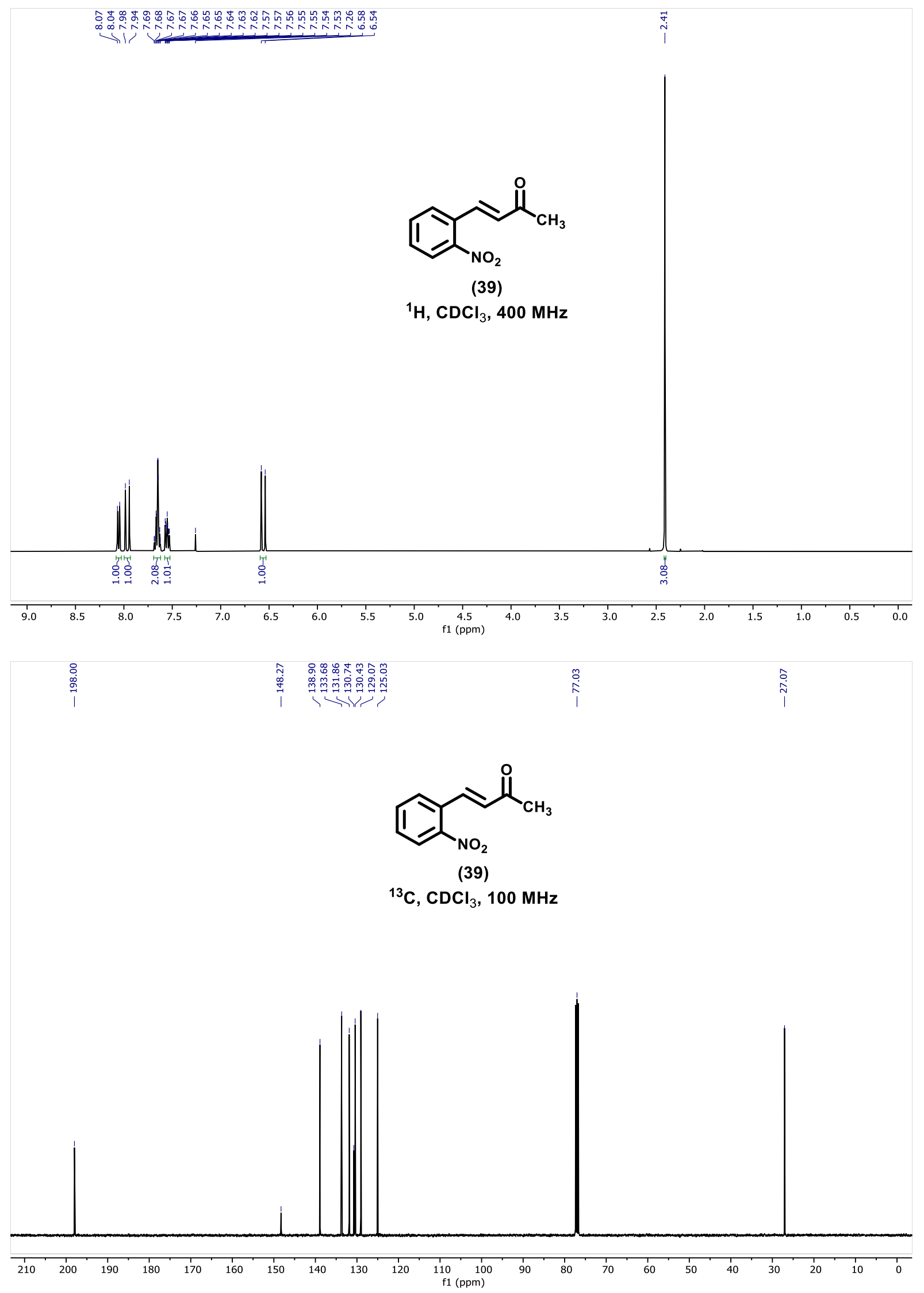




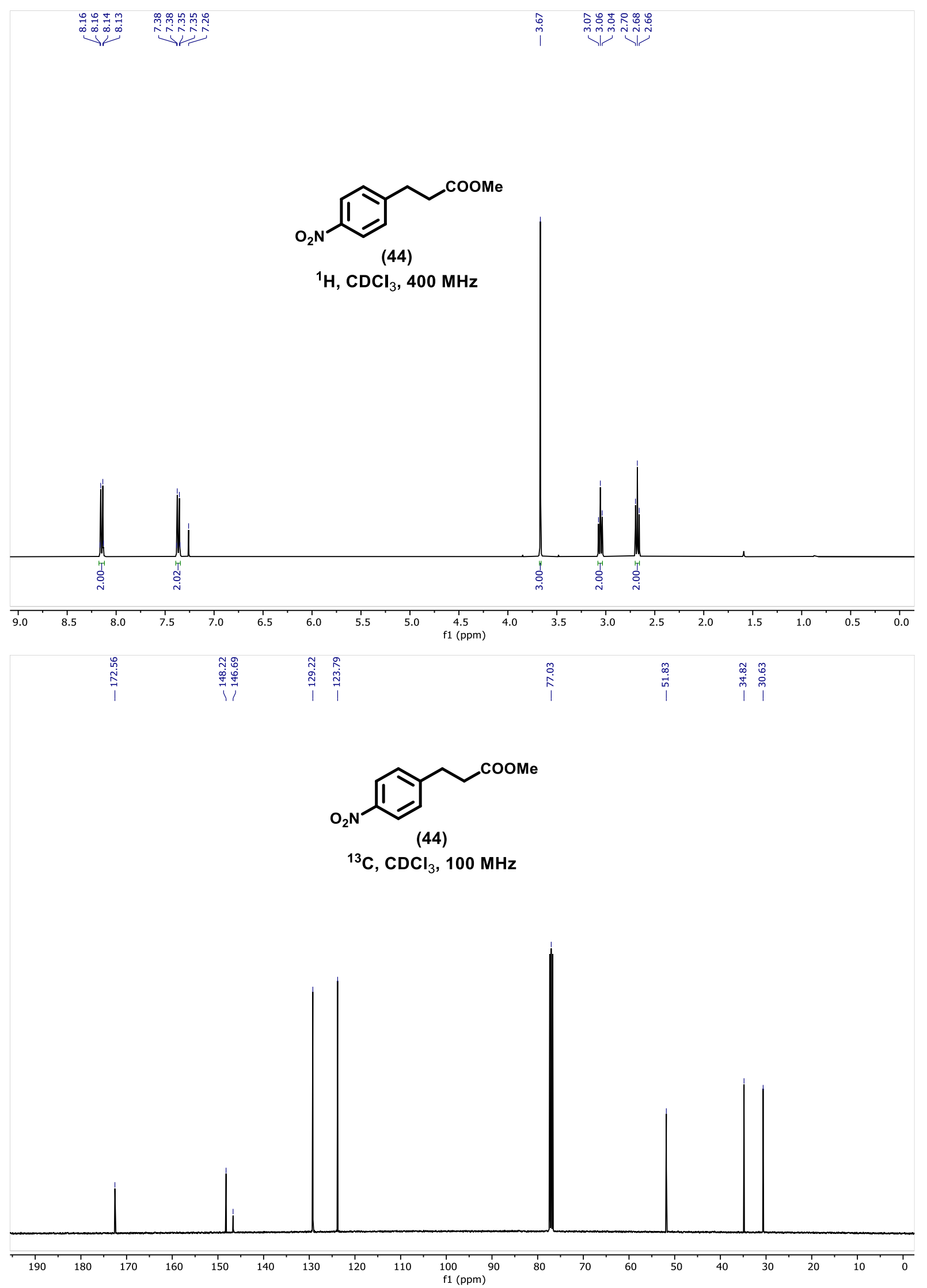

